archives-ouvertes

\title{
Kinetics of field-induced phase separation of a magnetic colloid under rotating magnetic fields
}

\author{
Maxime Raboisson-michel, Jordy Queiros Campos, S. Schaub, Andrey Yu. \\ Zubarev, Gregory Verger-dubois, Pavel Kuzhir
}

\section{To cite this version:}

Maxime Raboisson-michel, Jordy Queiros Campos, S. Schaub, Andrey Yu. Zubarev, Gregory Vergerdubois, et al.. Kinetics of field-induced phase separation of a magnetic colloid under rotating magnetic fields. Journal of Chemical Physics, American Institute of Physics, 2020, 153 (15), pp.154902. 10.1063/5.0023706 . hal-02975884

\section{HAL Id: hal-02975884 \\ https://hal.archives-ouvertes.fr/hal-02975884}

Submitted on 25 Dec 2020

HAL is a multi-disciplinary open access archive for the deposit and dissemination of scientific research documents, whether they are published or not. The documents may come from teaching and research institutions in France or abroad, or from public or private research centers.
L'archive ouverte pluridisciplinaire HAL, est destinée au dépôt et à la diffusion de documents scientifiques de niveau recherche, publiés ou non, émanant des établissements d'enseignement et de recherche français ou étrangers, des laboratoires publics ou privés. 


\title{
Kinetics of field-induced phase separation of a magnetic colloid under rotating magnetic fields
}

\author{
M. Raboisson-Michel ${ }^{1,2}$, J. Queiros Campos ${ }^{1}$, S. Schaub ${ }^{3}$, A. Zubarev ${ }^{4,5}$, G. Verger-Dubois², \\ P. Kuzhir ${ }^{*}$ \\ ${ }^{1}$ Université Côte d'Azur, CNRS UMR 7010, Institute of Physics of Nice, Parc Valrose, 06108 Nice, \\ France \\ ${ }^{2}$ Axlepios Biomedical - lere Avenue 5eme rue, 06510 Carros, France \\ ${ }^{3}$ Sorbonne University, CNRS, Developmental Biology Laboratory (LBDV), Quai de la Darse, 06234 \\ Villefranche-sur-Mer Cedex, France \\ ${ }^{4}$ Theoretical and Mathematical Physics Department, Institute of Natural Sciences and Mathematics, \\ Ural Federal University, Lenin Ave, 51, Ekaterinburg, 620083, Russia, \\ ${ }^{5}$ M.N. Mikheev Institute of Metal Physics of the Ural Branch of the Russian Academy of Sciences, \\ Ekaterinburg, Russia
}

\begin{abstract}
This paper is focused on the experimental and theoretical study of the phase separation of a magnetic nanoparticle suspension under rotating magnetic field in a frequency range, $5 \leq v \leq$ $25 \mathrm{~Hz}$, relevant for several biomedical applications. The phase separation is manifested through the appearance of needle-like dense particle aggregates synchronously rotating with the field. Their size progressively increases with time due to the absorption of individual nanoparticles (aggregate growth) and coalescence with neighboring aggregates. The aggregate growth is enhanced by the convection of nanoparticles towards rotating aggregates. The maximal aggregate length, $L_{\max } \propto v^{-2}$, is limited by fragmentation arising as a result of their collisions. Experimentally, aggregate growth and coalescence are occurring at a similar timescale, $\sim 1$ min, weakly dependent on the field frequency. The proposed theoretical model provides a semi-quantitative agreement with the experiments on average aggregate size, aggregation timescale and size distribution function, without any adjustable parameter.
\end{abstract}

\section{Introduction}

During the last two decades, magnetic nanoparticles received growing interest in regard of the numerous biomedical applications - see helpful reviews by Gupta and Gupta (2005), Tartaj et al. (2006). Some of them are related to the excitation of magnetic particles dispersed in biological fluids by alternating (oscillating or rotating) magnetic fields. Firstly, the detection of ferromagnetic hemozoin and $\beta$-hematin nanorods in blood samples by magneto-optical effects allows a more sensitive and swift detection of early stage malaria [Newmann et al. (2008)]. Secondly, magnetic iron oxide or cobalt nanorods could serve as a smart nano-platform for invitro immunoassays by monitoring the dynamics of nanorods with adsorbed target biomolecules under applied alternating magnetic fields [Schrittwieser et al. 2016; Schrittwieser et al. 2017; Newman and Matelon (2015)]. Similarly, intracellular viscoelasticity could be probed through the dynamics of chain-like clusters of the magnetized vesicles whose angular

\footnotetext{
${ }^{*}$ Corresponding author e-mail: pavel.kuzhir@univ-cotedazur.fr
} 
motion is induced by oscillating magnetic fields [Wilhelm et al. (2003); Berret (2016)]. Thirdly, magnetic nanoparticles of different shapes have been extensively tested for the destruction of tumors through mechanical action on cellular membranes under low-frequency magnetic fields [Wang et al. (2013); Leulmi et al. (2015); Chiriac et al. (2018)] or through magnetic hyperthermia under high-frequency fields [Perigo et al. (2015)]. Finally, blood clots in obstructed blood vessels can be non-invasively dissolved through the enhanced microcirculation of blood flow generated by rotating magnetic nanoparticles aggregates promoting either mechanical erosion [Gabayno et al. (2015)] or fast lysis of thrombi due to enhanced convection of thrombolytic drugs [Creighton (2012); Li et al. (2018)].

In most of the above-cited applications, magnetic nanoparticles are subject to field-induced phase separation manifested through the appearance of micron-sized particle aggregates with anisotropic shape. Such phase separation is a necessary phenomenon for some applications (probing of intracellular viscoelasticity, lysis of blood clots) and is a parasite phenomenon for other applications (immunoassays, hyperthermia). In any event, the knowledge of the aggregation timescale is of crucial importance for the successful realization of a given application. This requires a detailed study of the kinetics of the phase separation process.

Such kinetics is quite well documented in the case of a permanent magnetic field. Experimental studies by light scattering or direct optical visualization on 10-20 nm-sized nanoparticles revealed elongated scattered patterns ascribed to long drop-like aggregates whose growth with time was limited by lateral dipolar repulsion between them [Socoliuc and Bica (2001); Lashkar et al. (2010); Vinod and Philip (2018)]. This is in direct contradiction to the long-time observation by Swan et al. (2012) under microgravity conditions when all the aggregates coalesced into a single bulk phase. Similar experiments on superparamagnetic nanoclusters or nano-emulsion droplets of 30-200 nm-size range also revealed the appearance of long needle-like aggregates growing with time due to the absorption of single nanoclusters from the dilute phase surrounding the aggregates followed by the coalescence of aggregates due to dipolar attractions. This coalescence was however hindered at long times by dipolar repulsions [Socoliuc et al. (2013); Ezzaier et al. (2017); Ezzaier et al. (2018); Mohapatra and Philip (2019)]. From a theoretical perspective, the driving force of the field-induced phase separation is the supersaturation $\Delta=\varphi-\varphi^{\prime}$ defined as the difference of the nanoparticle volume fraction $\varphi$ of the phase separating colloid and $\varphi^{\prime}-$ the threshold volume fraction below which there is no phase separation. The term $\varphi^{\prime}$ is nothing but the equilibrium concentration of the dilute phase at the end of the phase separation process. In their works, Zubarev and Ivanov (1997) and Ivanov and Zubarev (1998) have considered the homogeneous nucleation and aggregate growth through the diffusion of nanoparticles $(15-20 \mathrm{~nm})$ towards the aggregates, followed by Oswald ripening allowing the formation of a bulk concentrated phase. They have established the scaling laws for the average aggregate volume at the aggregate growth stage, $\langle V\rangle \propto t^{7 / 4}$ and at the Oswald ripening stage, $\langle V\rangle \propto t^{7 / 6}$. Later on, Ezzaier et al. (2017) have modelled the kinetics of aggregation of relatively large superparamagnetic nanoclusters $(\sim 50$ $\mathrm{nm}$ ) and established that the diffusive flux still dominated over the magnetophoretic one for the aggregate growth stage, while aggregate coalescence on longer timescales conducted to a very 
fast aggregation, with $\langle V\rangle \propto t^{7 / 2}$ and dominated over the Oswald ripening in agreement with experiments.

A series of publications have been devoted to equilibrium structures and kinetics of phase separation in pulsed unidirectional fields with a rectangular waveform. Depending on the ratio between the "field on" and "field off" periods, needle-like ellipsoidal aggregates [Promislow and Gast (1997)], percolated or columnar structures [Swan et al. (2014)] or even branched structures perpendicular to the applied field [Kim et al. (2020)] have been observed and predicted through numerical simulations [Sherman and Swan (2016); Sherman et al. (2018)]. The average width $\langle s\rangle \propto t^{m}$ of these structures shows modest diffusion-limited growth in the beginning, with $m \approx 1 / 4-1 / 3$, followed by a faster growth due to coalescence, with $m \approx$ 3/2 [Swan et al. (2014); Kim et al. (2020)].

To the best of our knowledge, the kinetics of phase separation has never been studied in rotating magnetic fields, which offer a rich diversity of behaviors. Similar to their behavior in permanent field, the rotating field at relatively low frequency gather magnetic nanoparticles into elongated drop-like aggregates that rotate synchronously with the field [Sandre et al. (1999)]. However, the final state of magnetic colloid is considerably different. In a permanent field, all the aggregates coalesce into the bulk concentrated phase and the system settles in its equilibrium ground state with minimal energy [Swan et al. (2012)]. In a rotating field, the formation of the concentrated bulk phase seems to be hindered by hydrodynamic repulsion between rotating aggregates. Recently, Sitkuts et al. (2020) have observed that the magnetic droplets do not coalesce but organize themselves into a crystalline structure. They argue that a balance between attractive magnetic dipolar and repulsive hydrodynamic interactions is responsible for such non-equilibrium structuring. At higher frequencies of the rotating field, drop-like aggregates rotate asynchronously with the field, and, thanks to hydrodynamic interactions with the suspending liquid, their shape evolves into a bent rod, starfish or oblate ellipsoid depending on the field amplitude and frequency [Bacri et al. (1994); Cebers (2002); Lebedev et al. (2003)] but in any case, the collapse of all the aggregates into a single bulk phase has never been reported.

Kinetics of phase separation under rotating magnetic fields is expected to share some common features with kinetics in permanent fields, such as (a) fast nucleation at short times; (b) proportionality between the aggregate growth rate and supersaturation, $\dot{V} \propto \Delta$; and (c) coalescence of aggregates induced by the dipolar attraction between them. On the other hand, rotating magnetic fields drive the aggregates into rotation with respect to the suspending liquid, and the following qualitative differences with respect to the permanent field could be anticipated: (a) convective flux of nanoparticles towards the aggregates is likely to become significant during the aggregate growth stage, and (b) the aggregate growth and coalescence seem to be hindered by the hydrodynamic interactions which can even lead to aggregate fragmentation, not allowing the system to settle into the ground state with a single bulk phase. Quantitatively, the timescales of aggregate growth and coalescence are expected to be influenced by the field frequency and it is not a priori known whether the former will still be shorter than the latter. The above points related to aggregation in rotating magnetic fields require a detailed study. 
This paper is devoted to the kinetics of phase separation of a magnetic colloid in the particular case of a low frequency rotating magnetic field promoting elongated aggregates synchronously rotating with the field. In experiments, we use a slightly destabilized suspension of citrated iron oxide nanoparticles in water and follow the aggregation process by simple optical visualization. Evolution of the aggregate size, number density and volume fraction allows inspection of aggregate growth versus coalescence mechanisms. Along, we develop a theoretical model accounting for both these mechanisms based on the population balance equation by analogy with a more general phenomenon of condensation-driven aggregation of particles or liquid drops [Hassan and Hassan (2008)]. From a general perspective, our particular system exhibits behaviors common with another colloidal or molecular systems, such as (a) convective diffusion of nanoparticles on the aggregate surface similar to the particle deposition onto a collector surface during filtration process [van de Ven (1989)]; (b) coalescence and fragmentation of aggregates moving under the action of rotating field similar to coalescence and fragmentation of colloidal aggregates, liquid drops or bubbles in a multi-phase flow [Kolev (1993)]. From a practical approach, the present study is important for the successful development of the aforementioned biomedical applications in which nanoparticle aggregation plays a significant role.

The paper is organized as follows. The theoretical model is developed in Sec. II where the population balance equation is formulated and solved analytically for the aggregate size distribution. Experimental techniques are outlined in Sec. III. The model predictions are critically compared with the experimental results on integral characteristics (average aggregate size, number density and volume fraction of aggregates versus time) and the size distribution in Sec. IV. Conclusions are outlined in Sec. V.

\section{Theory}

\section{A. General framework}

Let us consider initially homogeneous suspension of superparamagnetic nanoparticles or nanoclusters (composed of superparamagnetic nanoparticles) dispersed in a Newtonian liquid of a viscosity $\eta_{0}$ at a volume fraction $\varphi_{0}$ and sandwiched between two horizontal planes separated by a relatively small distance $h(=200 \mu \mathrm{m}$ in our experiments). In the absence of the applied magnetic field, the suspension is supposed to be stable against aggregation. The homogeneous and circularly polarized rotating magnetic field of an amplitude $H_{0}$ and angular frequency $\omega$ is applied at the moment $t=0$. Above some critical values of $H_{0}$ and $\varphi_{0}$, magnetic attractive interactions between nanoparticles may induce a phase separation manifested through the appearance of needle-like aggregates, which in the low-frequency limit, keep their straight shape assimilated to a prolate ellipsoid with major and minor semi-axes denoted by $a$ and $b$, respectively, and rotating synchronously with the magnetic field [Sandre et al. (1999)]. The aggregates will certainly have unequal sizes but most of them usually have a high aspect ratio $r_{a}=a / b \gg 1$ and are supposed to respect a linear magnetization law with a constant magnetic susceptibility $\chi$ (defined in Appendix A) at typical magnetic fields $H_{0} \sim 10 \mathrm{kA} / \mathrm{m}$. Experiments show that at short times after the magnetic field application, all the aggregates move towards the same horizontal plane where they perform collective rotation. An ensemble of rotating aggregates is schematically shown in Fig. 1a. 

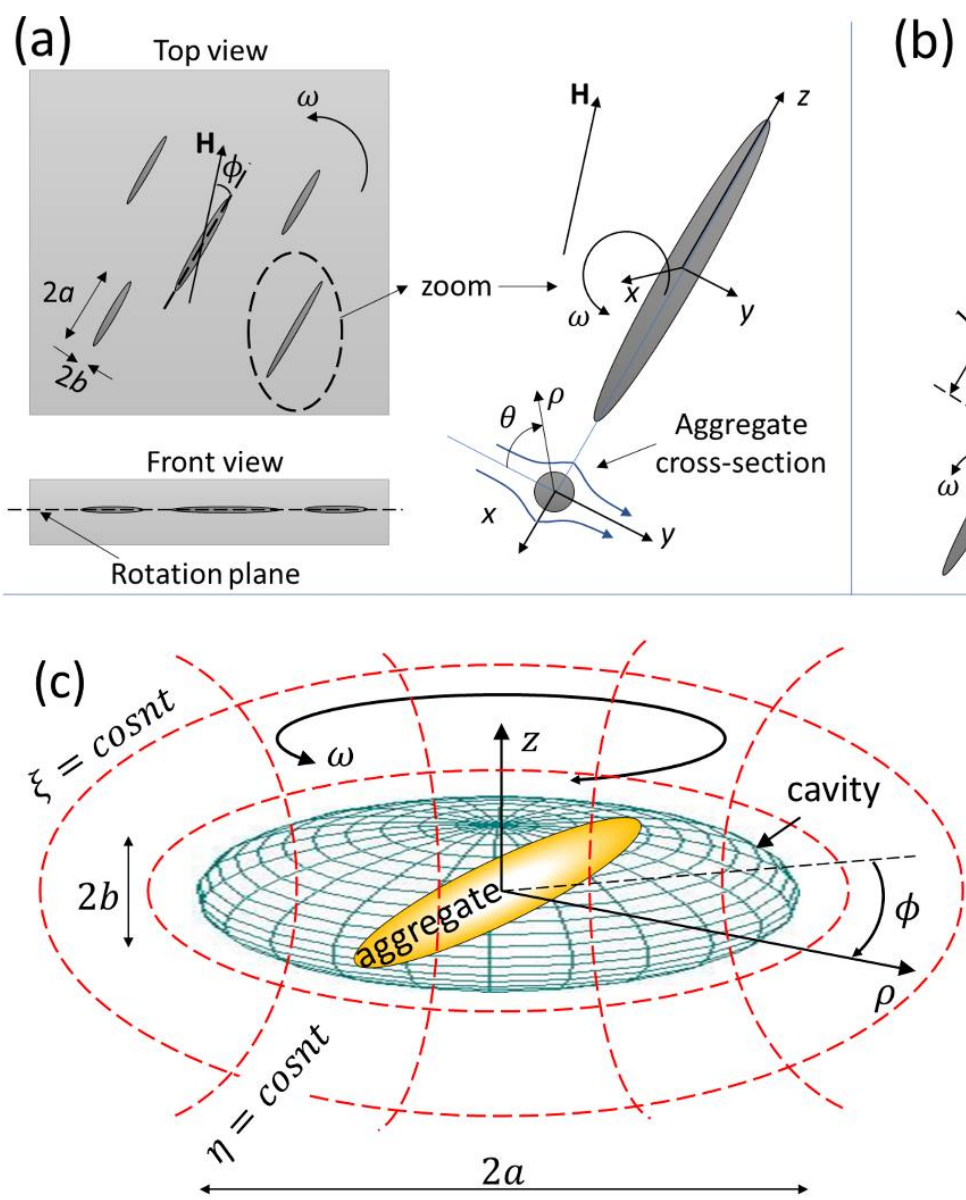

(b)

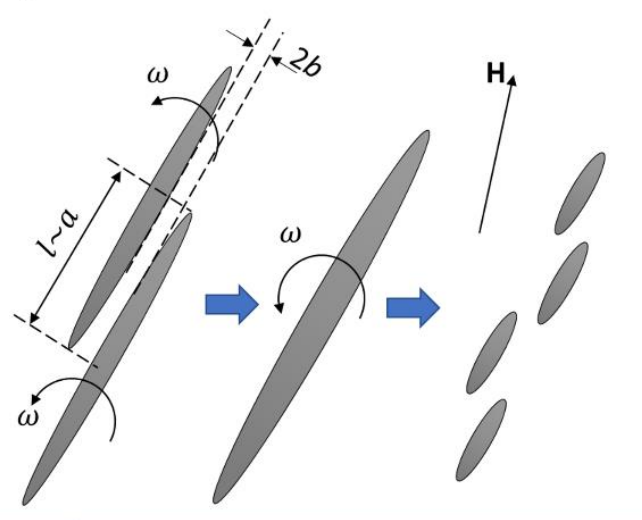

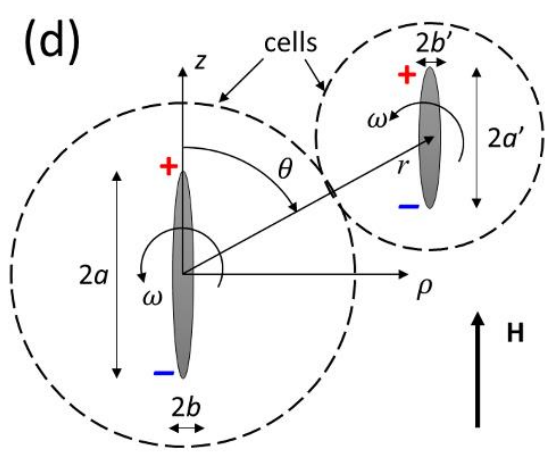

Fig. 1. Problem geometry. The ensemble of ellipsoidal aggregates involved in counterclockwise rotation by a rotating magnetic field is shown in (a). The aggregates make a weak angle $\phi \ll 1$ with the instantaneous direction of the applied field $\mathbf{H}$ (top view) and all of them rotate in a single horizontal plane between the channel walls (front view). A magnified view of a single aggregate is illustrated on the right of (a) showing a relative motion of the suspending liquid relatively to the aggregate and allowing calculation of the nanoparticle convective flux [Sec. II$\mathrm{D}$ and Appendix C]. Possible fragmentation scenario with the transient coalescence of two aggregates followed by splitting into several parts is depicted in (b). The cavity model allowing evaluation of the aggregate growth rate [Sec. II-D, Appendix D] is schematized in (c). The aggregates rotate inside an oblate ellipsoidal cavity swept by the aggregate rotational motion; the nanoparticle transport towards the aggregates is realized in two steps: (i) diffusion from infinity towards the cavity, and (ii) convective diffusion from the cavity to the aggregate across the diffusion boundary layer. The cell model used for evaluation of the coagulation kernel [Sec. II-E, Appendix E] is depicted in (d). The effective magnetic attraction between neighboring aggregates is treated as the interaction between equivalent "+" and "-" charges placed on the aggregate tips.

In the inertialess limit, typically holding in the low-frequency range, the phase lag $\phi$ between the instantaneous orientation of the major axis of the aggregates and the magnetic field vector is given by the equilibrium between hydrodynamic and magnetic torques by the following expression, adapted from [Sandre et al. (1999)]:

$$
\sin (2 \phi)=M a \approx \frac{8 r_{a}^{2} \eta_{0} \omega(2+\chi)}{3\left(\ln \left(2 r_{a}\right)-1 / 2\right) \mu_{0} H_{0}^{2} \chi^{2}},
$$

where $\mu_{0}=4 \pi \times 10^{-7} \mathrm{H} / \mathrm{m}$ is the magnetic permeability of vacuum and $M a$ is the Mason number defined as a characteristic ratio of the hydrodynamic-to-magnetic torque. Equation (1) shows that the aggregates can synchronously rotate with the field only at $M a \leq 1$. Furthermore, 
we consider the low Mason number limit, $M a \approx 2 \phi \ll 1$, respected in our experiments, for which possible effects of the phase lag $\phi$ on the aggregate magnetization and the supersaturation $\Delta$ can be neglected.

On the scale of the whole colloid, the evolution of aggregates is described by the distribution function $F(V, t)$ over the aggregate volumes $V$. The phase separation process is expected to be initiated by the nucleation, followed by the aggregate growth through absorption of individual nanoparticles, the aggregate coalescence under attractive dipolar interactions and possible fragmentation induced by hydrodynamic interactions or direct collisions between aggregates. It would be extremely difficult to handle simultaneously all these phenomena, but certain simplifications are hopefully possible. First, experiments show that the nucleation occurs at very short timescales and micron-sized aggregates appear at the times on the order of one second or less upon the magnetic field application [Ezzaier et al. (2017), see also videoa.avi in Supplemental Materials]. This allows the consideration of the nucleation process as infinitely fast compared to all the other stages of aggregation. We manage to evaluate an average size and the aspect ratio of the critical nuclei used as an initial condition for the subsequent aggregation stages. Secondly, the possible fragmentation of aggregates at the later stage is taken into account implicitly through the limitation of the aggregate growth and the coalescence rates vanishing as the aggregates average volume $\langle V\rangle$ achieves its maximal value dictated by the interplay between magnetic and hydrodynamic interactions. At such conditions, the aggregate size distribution function $F(V, t)$ must respect the population balance equation, in which the aggregate growth and the coalescence are explicitly taken into account, while the nucleation and fragmentation terms are omitted. This equation is similar to the one used in the condensation-driven coagulation model [Hassan and Hassan (2008)]:

$$
\begin{aligned}
& \frac{\partial F(V, t)}{\partial t}+\frac{\partial}{\partial V}(\dot{V} \cdot F(V, t))=\frac{1}{2} \int_{0}^{V} K\left(V^{\prime}, V-V^{\prime}\right) F\left(V-V^{\prime}, t\right) F\left(V^{\prime}, t\right) d V^{\prime}- \\
& F(V, t) \int_{0}^{\infty} K\left(V, V^{\prime}\right) F\left(V^{\prime}, t\right) d V^{\prime},
\end{aligned}
$$

with the distribution function normalized to the number of aggregates per unit volume, or shortly, the number density $n(t)$ :

$$
\int_{0}^{\infty} F(V, t) d V=n(t)
$$

The second term in the left-hand side of Eq. (2) corresponds to the aggregate growth mechanism with the growth rate $\dot{V} \equiv d V / d t$ and the two terms of the right-hand side correspond to the coalescence of aggregates with the coagulation kernel $K\left(V, V^{\prime}\right)$, which depends on attractive magnetic interactions between them. The diffusive term related to thermal fluctuations of the nanoparticle flux towards the aggregates is omitted - a conventional approximation in the theory of condensation valid for aggregate sizes larger than the size of the critical nuclei [Lifschitz and Pitajewski (1983)]. In the absence of coalescence terms, equation (2) reduces to classical Liouville equation often used for condensation phase transitions [Landau and Lifshitz (1980)], while in the absence of the convective (aggregate growth) term, equation (2) reduces to classical Smoluchowski equation used for irreversible aggregation in colloidal systems [Levich (1962)]. 
To proceed with this equation, we will first evaluate the average size of the critical nuclei (Sec. II-B). This evaluation will be sufficient for calculations of integral characteristics (average volume, number density and volume fraction of aggregates) independent of the precise shape of the size distribution of the critical nuclei. Then we will evaluate the maximal aggregate volume $V_{\max }$ (Sec. II-C) along with the aggregate growth rate $\dot{V}$ (Sec. II-D) and the coagulation kernel $K\left(V, V^{\prime}\right)$ (Sec. II-E) both depending on $V_{\text {max }}$. Finally, the analytical solution of the population balance equation (2) will be presented for both the integral characteristics (Sec. IIF) and the aggregate size distribution (Sec. II-G).

\section{B. Size of the critical nuclei}

In the polydisperse colloid, the nuclei can appear as microdroplets (composed of small nanoparticles) growing on the surface of large nanoparticles. This gives rise to the heterogeneous nucleation, as opposed to the homogeneous nucleation, when the nuclei appear in the bulk of the colloid. According to the classical nucleation theory, only nuclei larger than some critical size can cross the nucleation barrier and continuously grow [Lifschitz and Pitajewski (1983)]. Assuming the critical nuclei to be much larger than their condensation centers (largest nanoparticles in the suspension), the critical nuclei volume $V_{c}$ is shown to be nearly the same as in the case of the homogeneous nucleation and is determined at the local maximum of the minimal work of nucleus formation $\Delta G$ [Kalikmanov (2013)]:

$$
\left(\frac{\partial \Delta G}{\partial V}\right)_{V=V_{c}}=0 \text {. }
$$

Following Zubarev and Ivanov (1997), we assume that in the presence of an external magnetic field, the nuclei of a given volume $V$ have a shape of a prolate ellipsoid of an aspect ratio $r_{a}$, for which case the minimal work $\Delta G$ reads:

$$
\Delta G=-N \Delta \mu+\sigma S=-\frac{\varphi^{\prime \prime}}{V_{m}} V k_{B} T \ln \frac{\varphi_{0}}{\varphi^{\prime}}+\sigma V^{2 / 3} s\left(r_{a}\right)
$$

where $N$ is the number of individual nanoparticles of an average volume $V_{m}$ constituting the nucleus; $S$ and $\sigma$ are nucleus surface area and surface tension, respectively; $\Delta \mu$ is the difference of chemical potentials of nanoparticles far from the nucleus and at its surface; $k_{B} T \approx$ $4.1 \times 10^{-21} \mathrm{~J}$ is the thermal agitation energy at room temperature; $\varphi_{0}$ is the initial nanoparticle volume fraction before the phase separation; $\varphi^{\prime}$ and $\varphi^{\prime \prime}$ are equilibrium nanoparticle volume fractions in the dilute phase (in the vicinity of the nucleus) and the concentrated phase (inside the nucleus). The expression for the dimensionless surface area $s\left(r_{a}\right)$ for an elongated nucleus is provided in Appendix B [Eq. (B1c)]. Substituting Eq. (5) into Eq. (4), we get the relationship between the volume and aspect ratio of the critical nucleus, while the length of the critical nuclei is related to its volume and aspect ratio by the obvious geometrical expression:

$$
\begin{gathered}
V_{c}=\left[\frac{2 \sigma s\left(r_{c}\right) V_{m}}{3 \varphi^{\prime \prime} k_{B} T \ln \left(\varphi_{0} / \varphi^{\prime}\right)}\right]^{3} \propto\left(\frac{\sigma}{k_{B} T}\right)^{3}\left\langle d_{m}^{9}\right\rangle, \\
L_{c}=2\left[\frac{3 V_{c} r_{c}}{4 \pi}\right]^{1 / 3}
\end{gathered}
$$

The surface tension of the critical nuclei is taken to be the same as the surface tension of the supercritical aggregates. It depends on the amplitude $H_{0}$ of the applied magnetic field and 
its value was retrieved by fitting the experimental shape of the supercritical aggregates to the shape obtained theoretically by minimization of the aggregate free energy, as detailed in Appendix B. Energy minimization provides an expression [Eq. (B4)] for the aggregate (nucleus) volume as a function of the aspect ratio. Equalizing the right-hand parts of Eqs. (6a) and (B4) upon replacing $r_{a}$ by $r_{c}$, we arrive at a transcendental equation for the aspect ratio $r_{c}$ of the critical nuclei, which is solved numerically. The volume $V_{c}$ and the length $L_{c}$ of the critical nuclei are then evaluated by injecting numerical value of $r_{c}$ into Eqs. (6a) and (6b), respectively. The unknown equilibrium volume fractions $\varphi^{\prime}$ and $\varphi^{\prime \prime}$ are retrieved from experiments on the aggregation threshold (Sec. IV-B) and the aggregate translational motion under applied magnetic field gradient (Appendix A). According to Eq. (6a), the critical nucleus volume $V_{c} \propto$ $\left(\sigma V_{m} / k_{B} T\right)^{3} \propto\left(\sigma / k_{B} T\right)^{3} d_{m}^{9}$ is proportional to the $9^{\text {th }}$ power of the diameter $d_{m}$ of individual nanoparticles constituting the nuclei (the subscript " $m$ " comes from so-called "magnetic" diameter of nanoparticles found by magnetometry - see Sec. III-A). In the present case of polydisperse magnetic colloid, it seems reasonable to take a $9^{\text {th }}$ moment of the particle size distribution $\left\langle d_{m}^{9}\right\rangle$ [cf. Eq. (39) in Sec. III-A] for evaluation of $V_{c}$, putting $V_{m}^{3}=(\pi / 6)^{3}\left\langle d_{m}^{9}\right\rangle$ in Eq. (6a).

By deriving Eq. (6a), we supposed that the phase equilibrium on the aggregate surface is not affected by the flow generated by the aggregate rotation such that the critical nuclei size is found to be independent of the field frequency. This strong hypothesis will be carefully inspected and confirmed in Sec. IV-B in conjunction with an experimental phase diagram. The results on the critical nuclei size will be analyzed in Sec. IV-C.

\section{Maximal average aggregate volume}

Now we move to the opposite limit of the phase separation process where rotating aggregates become so long that they are subject to hydrodynamic interactions with neighboring aggregates. At first glance, the average aggregate length can be limited by geometric constraints, i.e. when the circles described by extremities of rotating aggregates start overlapping, in which case $n_{s} L_{\text {max }}^{2} \sim 1$, with $n_{s}$ - the number of aggregates per unit surface of the plane of their rotation. Experimentally, $L_{\max }$ is usually much less than the value predicted by geometric arguments.

On the other hand, an aggregate can be rapidly fragmented into two parts if at some moment the hydrodynamic force $F_{h}$ acting on the aggregate surface (in the hydrodynamic field created by the rotation of neighboring aggregates) becomes tensile and overcomes the surface tension force $F_{S}$ (acting as a compressive force tending to minimize the aggregate surface), as supposed by Zubarev et al. (2014) for magnetorheological suspensions under shear. The characteristic ratio of these forces reads: $F_{h} / F_{S}=\left(\eta_{0} \omega a^{2}\right) /(\sigma b)$ and is evaluated to be of the order of $F_{h} / F_{S} \sim 10^{-2}-10^{-1}$ in our experimental conditions. A precise calculation shows that the maximal aggregate length overcomes by two orders of magnitude the maximal length stemming from geometrical considerations. Therefore, we have to exclude this mechanism from consideration.

Alternatively, the aggregates can fragment after a direct collision between them. Strictly speaking, direct mechanical contacts are avoided by short-range lubrication interactions as long as the gap $h_{0}$ separating two approaching surfaces is larger than typical surface roughness, which in case of particle aggregates is on the order of magnitude of the average nanoparticle 
diameter $\left\langle d_{p}\right\rangle$ stemming from dynamic light scattering measurements (Sec. III-A). Collision dynamics of elongated aggregates is expected to be a quite complicated phenomenon involving non-trivial transient shapes, capillary waves and surface instabilities resulting in different outcomes, such as bouncing, coalescence, stretching or reflective separation (fragmentation), reported for droplet collisions [Qian and Law (1997)]. The detailed study of this dynamics is out of scope of the present work. Although, we can anticipate that longer aggregates colliding at higher relative speeds $(\sim \omega a)$ will be less stable to fragmentation than shorter ones by analogy with long liquid filaments forming during coalescence of two spherical drops and breaking up at high speeds because of surface instabilities. In our experiments, we observe that the collision between two long enough aggregates often produces coalescence on a very short timescale followed by the fragmentation of the new aggerate into three of four parts (Sec. IV-A, also see the video-b.mp4 in Supplemental materials). Furthermore, short-ranged hydrodynamic repulsion between aggregates during their collision certainly slows down their rotation and is expected to increase the phase lag $\phi$ between their major axis and the field. If the aggregates are long enough, the phase lag could overcome the critical value $\phi=\pi / 4$, above which synchronous rotation of two colliding aggregates is no longer possible, as mentioned below Eq. (1). Asynchronous rotation of the pair of colliding aggregates progressively increases the phase lag $\phi$ and when it achieves the value $\phi=\pi / 2$, the aggregate magnetization takes its minimal value ( $\sim 2 H_{0}$ as opposed to $\sim \chi H_{0}$ at $\phi=0$, with $\chi \gg 1$ ) for which the cohesive strength and surface tension of aggregates are expected to be minimal. This probably increases the probability of fragmentation. The fragmentation process is schematically presented in Fig. 1b.

For quantitative evaluation of the maximal aggregate size, we assume that during a collision, short-range lubrication interaction dominates over the hydrodynamic drug on rotating aggregates. Therefore, for these short times, we put the rate of energy dissipation, $w_{\text {diss }}$ through lubrication between laterally colliding aggregates equal to the power, $w_{m}$ injected to the aggregate by the rotating magnetic field. The rate of dissipation $w_{\text {diss }}$ is evaluated using the results of Christensen (1993) for two laterally approaching cylinders, while the power $w_{m}$ is a product of the magnetic torque $\Gamma_{m}$ [expression provided in Kuzhir et al. (2009)] taken at the transition between synchronous and asynchronous regimes $(\phi=\pi / 4)$ by the angular speed $\omega$ of aggregates equal to the angular frequency of the field:

$$
\begin{gathered}
w_{\text {diss }} \approx \frac{3}{4} \pi \eta_{0} a^{2} l \omega^{2}\left(\frac{b}{h_{0}}\right)^{3 / 2}, \\
w_{m}=\Gamma_{m} \omega=\frac{1}{2} \mu_{0} H_{0}^{2} \frac{\chi^{2}}{\chi+2} \sin (2 \phi) V \omega=\frac{2}{3} \pi \mu_{0} H_{0}^{2} \frac{\chi^{2}}{\chi+2} a b^{2} \omega,
\end{gathered}
$$

where $l \sim a$ is the longitudinal overlap between two colliding aggregates [Fig. 1b], taken to be on the order of the aggregate semi-length $a$. Equating right-hand parts of Eqs. (7a) and (7b), we arrive at the expression for the maximal average aggregate aspect ratio:

$$
r_{\text {max }} \approx\left(\frac{8}{9} \frac{\chi^{2}}{\chi+2} \frac{\mu_{0} H_{0}^{2}}{\eta_{0} \omega}\right)^{1 / 2}\left(\frac{h_{0}}{b}\right)^{3 / 4}
$$


where the gap $h_{0}$ between two aggregates can be considered as an adjustable parameter of the order of one or a few nanoparticle hydrodynamic diameters: $h_{0} \sim\left\langle d_{p}\right\rangle$. In this formulation, the maximal aspect ratio is only defined by a collision between two isolated aggregates. In reality, it is expected to be defined by the interplay of coalescence and fragmentation events and their frequency. It could thus depend on the volume fraction $\Phi$ of aggregates in the suspension. However, the volume fraction $\Phi$ in our model and experiments shows only a moderate change with the field frequency $\omega$ [Sec. II-F, IV-D] and, within the range of our experimental parameters, we consider $r_{\max }$ to be independent of $\Phi$.

The maximal average aggregate length and volume are then found as

$$
\begin{aligned}
& L_{\max }=2 b r_{\max }, \\
& V_{\text {max }}=\frac{4}{3} \pi b^{3} r_{\text {max }} .
\end{aligned}
$$

The two remaining unknowns in Eqs. (8) and (9), the aggregate half-thickness $b$ is found to be nearly independent of the aggregate volume and is determined in Appendix B, while the aggregate magnetic susceptibility $\chi$ is found in Appendix A from the experiments on the translational motion of aggregates in the gradient magnetic field. The results of Eq. (9a) are compared with experiments in Sec. IV-C.

\section{Aggregate growth rate}

Once the critical nuclei appear, they continuously grow due to absorption of individual nanoparticles from the dilute phase surrounding them. The transport of individual nanoparticles towards the aggregates arises thanks to three possible mechanisms as follows: (a) diffusion due to the difference of nanoparticle volume fraction far from the aggregates $(\varphi)$ and in the vicinity of their surface $\left(\varphi^{\prime}\right)$, referred to as supersaturation $\Delta=\varphi-\varphi^{\prime}$; (b) migration due to magnetic attraction of nanoparticles to the aggregate, referred to as magnetophoresis; and (c) convection when the rotating aggregate "collects" nanoparticles from the macroscopically quiescent dilute phase; in the reference frame rotating with the aggregate, the nanoparticles are convected towards the aggregate by the flow of the suspending liquid generated by the aggregate rotation. The analysis shows that in the considered high aspect ratio limit, $r_{a} \gg 1$, the magnetic field distribution around the aggregates is nearly homogeneous, except for the regions near the aggregate tips, and the magnetophoretic flux gives an insignificant contribution (logarithmic with the magnetic field intensity) to the aggregate growth rate for the considered range of dipolar coupling parameters $\lambda_{d}=m_{p}^{2} /\left(4 \pi \mu_{0} k_{B} T d_{p}^{3}\right) \sim 1-10$, where $m_{p}$ is the nanoparticle magnetic moment [Ezzaier et al. (2017)]. Thus, the magnetophoretic flux can be safely neglected. Convective flux is compared to the diffusive flux through the Peclet number, defined for rotating aggregates with a linear velocity of its tip $\sim \omega a$, as follows:

$$
P e=\frac{2 b \omega a}{D_{p}}
$$

where $D_{p}=3 \pi \eta_{0} d_{p} /\left(k_{B} T\right)$ is the Brownian diffusivity of individual nanoparticles. The Peclet number takes the values $P e \sim 10^{2}-10^{3}$ in our experimental conditions, showing that the convective transport dominates over the diffusive one, except for a thin diffusive boundary 
layer close to the aggregate surface where the suspending liquid velocity takes small values in the reference frame of the aggregate due to non-slip hydrodynamic boundary condition. At such condition, we can apply the Levich's boundary layer approach developed for ion or small particle deposition onto the surface of large particles [Levich (1962)].

In the frames of this approach, we are looking for the volume flux $J_{V}$ of nanoparticles towards the aggregate, which is related to the aggregate growth rate $\dot{V}$ through the following obvious expression:

$$
J_{V}=\varphi^{\prime \prime} \dot{V}
$$

with the known nanoparticle volume fraction inside the aggregates $\varphi^{\prime \prime} \gg \varphi^{\prime}$ defined in experiments (Appendix A). The problem geometry is schematically presented on the magnified view of a single aggregate on the right of Fig. 1a.

Let us introduce the Cartesian reference frame Oxyz rigidly attached to the aggregate with the origin $O$ placed into the aggregate geometric center and $z$-axis oriented along the major aggregate axis. The aggregate rotates with an angular frequency $\omega$ around the $x$-axis. The analysis shows that for a long aggregate, $r_{a} \gg 1$, in the vicinity of the aggregate surface, the longitudinal $z$ component of the velocity is much smaller than the transverse $x$ and $y$ components. At such condition, the nanoparticle capture by unit aggregate length can be approximated as a convective diffusion of particles towards a cylinder placed into a homogeneous flow field with the fluid velocity at the infinity equal to $u_{\infty}=\omega z$ at a given axial position $z$, and with the volume flux $j_{V}$ per unite aggregate length given by the following expression [Adamczyk and van de Ven (1981)]:

$$
\begin{aligned}
& j_{V}=c A_{f}^{1 / 3} D_{p} P e_{z}^{1 / 3} \Delta, \\
& A_{f} \approx \frac{1}{2\left(\ln \left(2 r_{a}\right)-1 / 2\right)} \frac{\hat{r}^{2}}{\hat{r}^{2}+\hat{z}^{2} / r_{a}^{2}},
\end{aligned}
$$

where $c \approx 3.644 ; A_{f}$ is the hydrodynamic coefficient derived in Appendix $\mathrm{C}$ and coming from the velocity field in the vicinity of the rotating aggregate; $P e_{z}=P e \cdot \hat{r} \hat{z}$ is the local Peclet number at a given position $z$, related to the global Peclet number $P e$ given by Eq. (10); $\hat{r}=r / b$ and $\hat{z}=z / a$ are dimensionless radial and axial coordinates of the aggregate surface, related through $\hat{r}^{2}+\hat{z}^{2}=1$. The volume flux $J_{V}$ is obtained by integration of the linear flux density $j_{V}$ over the aggregate length:

$$
J_{V}=2 a \int_{0}^{1} j_{V} d \hat{z}=\frac{k}{\left(\ln \left(2 r_{a}\right)-1 / 2\right)^{1 / 3}} D_{p} P e^{1 / 3} \Delta
$$

with $k \approx 1.931$.

The particle volume flux evaluated by Eq. (13) is likely overestimated because of the following reason. When the aggregate rotates under an applied rotating magnetic field, it absorbs some part of neighboring single nanoparticles. At each new period of its rotation, the aggregate can encounter fewer and fewer nanoparticles. Thus, the convective flux can be 
substantially reduced a few spins after the beginning of the rotation. Particle transport in this situation can be evaluated using the following simple model. During its rotation, the aggregate sweeps a volume (hereinafter called a cavity) having a shape of an oblate ellipsoid formed by the revolution of the outer border of the aggregate's boundary layer, as shown schematically in Fig. 1c. Neglecting boundary layer thickness, the cavity has two major semi-axes equal to the aggregate half-length $a$ and the minor semi-axis equal to the aggregate half-thickness $b$.

We suppose that, inside the cavity, the particle transport is convection-diffusive and respects the boundary layer approach [Eq. (13)], with the only difference being that the average nanoparticle volume fraction inside the cavity (excluding the aggregate with its boundary layer) takes some unknown value $\varphi_{c}$, intermediate between the volume fractions in the vicinity of the aggregate surface $\left(\varphi^{\prime}\right)$ and at the infinity from the cavity $(\varphi)$, so that $\varphi^{\prime}<\varphi_{c}<\varphi$. We further suppose that the nanoparticles outside the cavity enter inside the cavity by diffusion thanks to the concentration difference $\varphi-\varphi_{c}$. The diffusive volume flux $J_{V 1}$ towards the cavity is given by the following expression, developed in Appendix D:

$$
J_{V 1}=8 D_{p} a\left(\varphi-\varphi_{c}\right)
$$

while the convection-diffusive flux $J_{V 2}$ towards the aggregates inside the cavity is given by Eq. (13), in which the supersaturation $\Delta$ has to be replaced by $\left(\varphi_{c}-\varphi^{\prime}\right)$. In a conventional approximation of quasi-stationary particle transport, both fluxes $J_{V 1}$ and $J_{V 2}$ are the same. Eliminating $\varphi_{c}$, we arrive at the following expression for the volume particle flux, which accounts for both the emptying of the cavity through particle absorption by the rotating aggregate and the filling of the cavity by particle diffusion towards the cavity:

$$
J_{V}=J_{V 1}=J_{V 2}=\left[\frac{8\left(\ln \left(2 r_{a}\right)-1 / 2\right)^{1 / 3}}{k P e^{1 / 3}}+1\right]^{-1} 8 D_{p} a \Delta .
$$

This last equation shows that at Peclet number limit $P e^{1 / 3} \gg 8\left(\ln \left(2 r_{a}\right)-1 / 2\right)^{1 / 3} / k \gg 1$ corresponding to high enough excitation frequency $\omega$, the particle flux reduces to Eq. (14) where $\left(\varphi-\varphi_{c}\right)$ has to be replaced by $\Delta$. The particle transport is dominated by diffusion towards the cavity because the aggregates rotate fast enough and rapidly empty the cavity such that the volume fraction $\varphi_{c}$ in the cavity approaches the equilibrium value $\varphi^{\prime}$. In the limit $1 \ll P e^{1 / 3} \ll 8\left(\ln \left(2 r_{a}\right)-1 / 2\right)^{1 / 3} / k$, corresponding to lower frequencies $\omega$, the particle flux reduces to Eq. (13). The transport is dominated by convective diffusion towards the aggregate, while the cavity has enough time to be filled by nanoparticles from outside during the aggregate rotation period. In our experimental conditions, we are at an intermediate situation, so the complete expression (15) for the particle flux has to be used.

To get the aggregate growth rate $\dot{V}$ as a function of the aggregate volume $V$ only, we have to express its geometrical characteristics $a, b$ and $r_{a}=a / b$ through $V$. This is done in Appendix $\mathrm{B}$ by minimization of free energy of a single aggregate of a given volume $V$ with respect to its aspect ratio. As mentioned in Sec. II-C, in our experimental conditions, the aggregate halfthickness $b$ appears to be nearly independent of the volume [Eq. (B6)] and approximate expressions for $a(V, b)$, and $r_{a}(V, b)$ can be easily obtained from the aggregate volume $V=$ $4 \pi a b^{2} / 3$ : 


$$
a=\frac{3 V}{4 \pi b^{2}}, \quad r_{a}=\frac{3 V}{4 \pi b^{3}} .
$$

Combining Eqs. (10), (11), (15), (16) and neglecting logarithmic variation of the aspect ratio setting it equal to the maximal value [Eq. (8)], $r_{a} \approx r_{\max }$, we arrive at the following expression for the aggregate growth rate:

$$
\begin{aligned}
& \dot{V} \approx\left[\frac{\alpha}{\left(V / V_{\max }\right)^{1 / 3}}+1\right]^{-1} \frac{6 D_{p} V}{\varphi \prime \prime \pi b^{2}} \Delta, \\
& \alpha=\frac{8\left(\ln \left(2 r_{\max }\right)-1 / 2\right)^{1 / 3}}{k P e_{\max }^{1 / 3}}, \quad P e_{\max }=\frac{3 V_{\max } \omega}{2 \pi D_{p} b},
\end{aligned}
$$

where $P e_{\text {max }}$ is the Peclet number for the aggregates of the maximal volume given by Eqs. (8), (9b).

As the aggregates absorb nanoparticles from the surrounding dilute phase, the particle volume fraction in the dilute phase, and consequently, the supersaturation $\Delta$ progressively decreases with time. The supersaturation can be expressed through the average aggregate volume $\langle V\rangle$ using the particle volume conservation condition $\varphi(1-\Phi)+\varphi^{\prime \prime} \Phi=\varphi_{0}$, where $\Phi$ is the volume fraction of aggregates defined as the ratio of the volume occupied by aggregates to the volume of the whole suspension. At low concentrations of the colloid, $\varphi_{0} \ll 1$ and at a typical internal volume fraction of aggregates $\varphi^{\prime \prime} \sim 0.3$ [cf. Appendix A], the strong inequality $\Phi \ll 1$ holds. With this in mind, and taking into account that $\Phi=n\langle V\rangle$, we get the following approximate expression for the supersaturation:

$$
\Delta=\varphi-\varphi^{\prime}=\Delta_{0}-\varphi^{\prime \prime} n\langle V\rangle
$$

where $\Delta_{0}=\varphi_{0}-\varphi^{\prime}$ is the initial supersaturation and $n$ is the number of aggregates per unit volume (number density).

Equation (18) shows that, in absence of hydrodynamic interactions and coalescence or fragmentation events (implying constant number density $n=$ const), the aggregates grow until their average volume achieves the maximal value $V_{e q}=\Delta_{0} /\left(\varphi^{\prime \prime} n\right)$, when the system tends to thermodynamic equilibrium and the supersaturation tends to zero. In these conditions, the supersaturation can be written as: $\Delta=\Delta_{0}\left(1-\langle V\rangle / V_{e q}\right)$. In reality, because of hydrodynamic interactions and collisions between rotating aggregates, they fragment, and their growth is limited by the maximal average volume $V_{\max }<V_{\text {eq }}$ evaluated in Sec. II-C [Eqs. (8), (9b)]. This means that at the end of the phase separation under rotating fields, the supersaturation will not achieve zero value. However, the aggregate growth (due to remaining supersaturation $\Delta \neq 0$ and possible coalescence) is expected to be counterbalanced by fragmentation of aggregates. The simplest way to account for fragmentation is to require zero growth rate $\dot{V}$ as the average volume $\langle V\rangle$ achieves its maximal value $V_{\text {max }}$. It can be done by replacing $V_{e q}$ by $V_{\text {max }}$ in the expression for $\Delta$, which after replacement in Eq. (17a) gives the final expression for the aggregate growth rate:

$$
\dot{V} \approx\left[\frac{\alpha}{\left(V / V_{\max }\right)^{1 / 3}}+1\right]^{-1} \frac{6 D_{p} V}{\varphi \prime \prime \pi b^{2}} \Delta_{0}\left(1-\frac{\langle V\rangle}{V_{\max }}\right)
$$


The magnitude $\dot{V}$ depends on the magnetic field frequency $\omega$ intervening into the constant $\alpha$ [Eq. (17b)] and the maximal average volume $V_{\max }$ [Eqs. (8), (9b)], as well as on the magnetic field amplitude $H_{0}$ intervening into $V_{\max }$ and initial supersaturation $\Delta_{0}$ [see the phase diagram on Fig. 5].

\section{E. Coagulation kernel}

The aggregates of a supercritical volume, $V>V_{c}$ can also coalesce thanks to attractive dipolar interactions at the same time as they grow by absorption of individual nanoparticles. The coalescence rate is expressed through so-called coagulation kernel $K\left(V, V^{\prime}\right)$, which in general depends on volumes $V$ and $V^{\prime}$ of two merging aggregates and is related to the magnetophoretic flux $J_{V \prime \rightarrow V}$ of the aggregates $V^{\prime}$ towards a target aggregate $V$ by the following expression:

$$
J_{V \prime \rightarrow V}=K\left(V, V^{\prime}\right) n_{V \prime}
$$

where $n_{V^{\prime}}=F\left(V^{\prime}, t\right) d V^{\prime}$ is the number density of aggregates $V^{\prime}$ in the suspension.

This flux is evaluated in Appendix E based on the cell model [Fig. 1d], and explicit expression [Eqs. E4] for the coagulation kernel is provided. The analysis shows that in the considered ranges of the aggregate aspect ratios $\left(r_{a}=5-50\right)$ and size ratios $\left(V / V^{\prime} \approx r_{a} / r_{a}{ }^{\prime}=\right.$ $1 / 10-10)$, as well as at a moderate increase of the volume fraction $\Phi$ of the aggregates with the time (Sec. IV-D, Fig. 8), the coagulation kernel varies maximum by $\sim \pm 20 \%$ with respect to some average value. Such variations are rather inessential as compared to those observed in various coagulation processes such as shear-induced coagulation, branched polymerization, etc. [Aldous (1999)]. It is, therefore, reasonable to consider the coagulation kernel to be constant: $K(V, V) \approx$ const $\equiv K_{0}$, where $K_{0}$ is hereinafter referred to as coagulation constant. For definiteness, we take the value of $K_{0}$ at the aggregate aspect ratio equal to half of its maximal average value imposed by hydrodynamics [Eq. (8)] and at the aggregate volume fraction $\Phi$ equal to the known initial value $\Phi_{0}$. Thus, $K_{0}$ is calculated by Eqs. (E4) in which the aspect ratio is set to $r_{a}=r_{a}^{\prime}=r_{\max } / 2$ and $\Phi$ is replaced by $\Phi_{0}$.

Aggregate coalescence with constant coagulation kernel is expected to lead to indefinite aggregate growth. However, fragmentation induced by hydrodynamic interactions must limit this growth to the maximal average volume evaluated by Eqs. (8), (9b). The simplest way to handle this problem without introducing fragmentation kernels is to impose a progressive decrease of the coagulation constant with an increasing average aggregate volume $\langle V\rangle$ until $K=0$ at $\langle V\rangle \rightarrow V_{\text {max }}$. Among different possibilities, this can be done by multiplying the coagulation constant by $1-\langle V\rangle / V_{\max }$. The expression for the modified coagulation constant depending on time and implicitly accounting for fragmentation reads:

$$
K(t)=K_{0}\left(1-\frac{\langle V\rangle}{V_{\max }}\right)
$$

In summary, the coagulation kernel $K\left(V, V^{\prime}\right)$ in the population balance equation [Eq. (2)] has to be replaced by the time-dependent coagulation constant $K(t)$ which, according to Eq. (E4a) scales as $K(t) \sim \mu_{0} \chi^{2} H_{0}^{2} b^{2} h / \eta_{0}$. 


\section{F. Integral characteristics}

Having defined the aggregate growth rate [Sec. II-D, Eq. (19)] and the coagulation kernel [Sec. II-E, Appendix E, Eq. (21)], we can proceed with the solution of the population balance equation [Eq. (2)], which can be rewritten in the following dimensionless form:

$$
\begin{aligned}
& \frac{\partial \psi(v, \tau)}{\partial \tau}+(1-\langle v\rangle) \frac{\partial}{\partial v}[g(v) \psi(v, \tau)]=\frac{1}{2} \kappa_{0}(1-\langle v\rangle) \int_{0}^{v} \psi\left(v-v^{\prime}, \tau\right) \psi\left(v^{\prime}, \tau\right) d v^{\prime}- \\
& \kappa_{0}(1-\langle v\rangle) \psi(v, \tau) \int_{0}^{\infty} \psi\left(v^{\prime}, \tau\right) d v^{\prime}, \\
& g(v)=\left[\alpha v^{-4 / 3}+v^{-1}\right]^{-1},
\end{aligned}
$$

introducing the following dimensionless quantities: $v=V / V_{\max }$ and $\langle v\rangle=\langle V\rangle / V_{\max }$ for the aggregate volume, $\psi(v, \tau)=\left(V_{\max }^{2} / \Phi_{0}\right) F(V, t)$ for the size distribution function, $\tau=t / t_{d}$ for the time and $\kappa_{0}=t_{d} / t_{c}$ for the ratio of the diffusion and coalescence timescales given by the following expressions:

$$
t_{d}=\frac{\pi b^{2} \varphi \prime \prime}{6 D_{p} \Delta_{0}}, \quad t_{c}=\frac{V_{\max }}{K_{0} \Phi_{0}} .
$$

The parameter $\kappa_{0}$ allows switching between aggregate growth mechanism at $\kappa_{0} \rightarrow 0$ and coalescence mechanism at $\kappa_{0} \rightarrow \infty$, while both mechanisms coexist at intermediate values $\kappa_{0}=$ $O$ (1) relevant to our experiments. Analytical solution of Eq. (22a) does not exist for the function $g(v)$ given by Eq. (22b). However, at the values $\alpha \leq 1$, relevant for our experiments, within the maximal error of $6 \%$, the function $g(v)$ can be replaced by its linearized form:

$$
g(v) \approx \frac{v}{1+\alpha} \equiv \beta v,
$$

with $\beta=1 /(1+\alpha)$ and $\alpha$ given by Eq. (17b) as a function of the Peclet number. Using Eq. (24), and introducing a change of variables $(1-\langle v\rangle) d \tau \equiv d \Theta$, the population balance equation takes the following approximate form:

$\frac{\partial \psi(v, \Theta)}{\partial \Theta}+\beta \frac{\partial}{\partial v}[v \psi(v, \Theta)]=\frac{1}{2} \kappa_{0} \int_{0}^{v} \psi\left(v-v^{\prime}, \Theta\right) \psi\left(v^{\prime}, \Theta\right) d v^{\prime}-\kappa_{0} \psi(v, \Theta) \int_{0}^{\infty} \psi\left(v^{\prime}, \Theta\right) d v^{\prime}$.

This equation has a similar mathematical structure that the one of dropwise condensation [Cueille and Sire (1997)], except for the dimensionless time $\tau$ replaced in our case by the variable $\Theta$, which measures the dimensionless time in a compressed timescale allowing for limitation of the aggregate growth. Multiplying Eq. (25) by $v^{j}, j=0,1$ and integrating over $v$, taking into account that $v^{j} \psi \rightarrow 0$ at $v \rightarrow \infty$, we get two independent ordinary differential equations for the $0^{\text {th }}$ and the $1^{\text {st }}$ moments $M_{j}(\Theta)=\int_{0}^{\infty} \psi(v, \Theta) v^{j} d v(j=0,1)$ of the size distribution function $\psi(v, \Theta)$, as follows:

$$
\frac{d M_{0}}{d \Theta}=-\frac{1}{2} \kappa_{0} M_{0}^{2}, \quad \frac{d M_{1}}{d \Theta}=\beta M_{1} .
$$

The solution of these equations at appropriate initial conditions allows finding the integral characteristics of phase separation, i.e. the aggregate number density $n$, the volume fraction of 
aggregates $\Phi$ and the dimensionless aggregate average volume $\langle v\rangle$ as a function of the reduced dimensionless time $\Theta$ :

$$
\begin{aligned}
& \frac{n(\Theta)}{n_{0}}=v_{0} M_{0}(\Theta)=\left[1+\frac{\kappa_{0} \Theta}{2 v_{0}}\right]^{-1}, \\
& \frac{\Phi(\Theta)}{\Phi_{0}}=M_{1}(\Theta)=e^{\beta \Theta}, \\
& \langle v\rangle(\Theta)=\frac{M_{1}(\Theta)}{M_{0}(\Theta)}=v_{0} e^{\beta \Theta}\left[1+\frac{\kappa_{0} \Theta}{2 v_{0}}\right],
\end{aligned}
$$

where $n_{0}, \Phi_{0}$ and $v_{0}$ are initial values of the number density, volume fraction of aggregates and dimensionless average volume, respectively. The initial volume can be evaluated as the dimensionless average volume of critical nuclei: $v_{0}=V_{c} / V_{\text {max }}$ [Eqs. (6a), (9b)]. One of the two parameters, $n_{0}$ or $\Phi_{0}$ is supposed to be known from experiments and the remaining parameter is found through the relationship $n_{0}=\Phi_{0} / V_{c}$. Finally, the dimensionless time $\tau$ is obtained from the reduced time $\Theta$, using Eq. (27c):

$$
d \tau=\frac{d \Theta}{1-\langle v\rangle} \Rightarrow \tau(\Theta)=\int_{0}^{\Theta} \frac{d \Theta \prime}{1-v_{0} e^{\beta \Theta \prime}\left[1+\kappa_{0} \Theta^{\prime} /\left(2 v_{0}\right)\right]},
$$

where the integral is evaluated numerically.

Since the dimensionless average volume is limited from above by $\langle v\rangle=1$ (corresponding to $\langle V\rangle=V_{\max }$ ), the reduced time cannot overcome a maximal value $\Theta_{\max }$ coming from the solution of the following transcendental equation:

$$
v_{0} e^{\beta \Theta_{\max }}\left[1+\frac{\kappa_{0} \Theta_{\max }}{2 v_{0}}\right]=1 .
$$

In summary, time dependencies of the integral characteristics of field-induced phase separation are evaluated in a parametric form with the help of Eqs. (27) and (29), using the reduced time $\Theta$ as a parameter and varying its value from 0 to $\Theta_{\max }$. These dependencies will be compared with experimental ones in Sec. IV-D. Notice that under considered approximation $g(v) \propto v$ [Eq. (24)], equivalent to the linear aggregate growth rate, $\dot{V} \propto V \Delta$, the integral characteristics depend only on the initial dimensionless average volume $v_{0}$ but are fully independent of the shape of the initial size distribution $\psi(v, 0)$. This has allowed us to find them without solving the population balance equation (25).

\section{G. Size distribution}

The size distribution function $\psi(v, \Theta)$ can be obtained by solution of Eq. (25) at some known initial size distribution $\psi(v, 0) \equiv \psi_{0}(v)$. Following Cueille and Sire (1997) and Hassan and Hassan (2008), applying the Laplace transform to the distribution function, $\Psi(p, \Theta)=$ $\mathcal{L}[\psi(v, \Theta)]=\int_{0}^{\infty} e^{-p v} \psi(v, \Theta) d v$ (with $p$ being a complex frequency parameter), the integrodifferential equation (25) is transformed into the following partial differential equation for $\Psi(p, \Theta)$ :

$$
\frac{\partial \Psi(p, \Theta)}{\partial \Theta}-\beta p \frac{\partial \Psi(p, \Theta)}{\partial p}=\frac{1}{2} \kappa_{0} \Psi^{2}(p, \Theta)-\kappa_{0} \Psi(0, \Theta) \Psi(p, \Theta), \quad \Psi(0, \Theta)=M_{0}(\Theta),
$$


whose analytical solution, obtained by the method of characteristics, reads:

$\Psi(p, \Theta)=\frac{(1-\vartheta)^{2} \Psi_{0}(p, \Theta)}{1-v_{0} \vartheta \Psi_{0}(p, \Theta)}, \Psi_{0}(p, \Theta)=\int_{0}^{\infty} e^{-p v \exp (\beta \Theta)} \psi_{0}(v) d v, \quad \vartheta=\frac{\kappa_{0} \Theta /\left(2 v_{0}\right)}{1+\kappa_{0} \Theta /\left(2 v_{0}\right)}$

For comparison with our experiments, it is more convenient to introduce the dimensionless distribution function $f(v, \Theta)$ with its $0^{\text {th }}$ and $1^{\text {st }}$ moments defined as follows:

$$
f(v, \Theta) \equiv \frac{\psi(v, \Theta)}{M_{0}(\Theta)}, \quad \int_{0}^{\infty} f(v, \Theta) d v=1, \quad \int_{0}^{\infty} f(v, \Theta) v d v=\langle v\rangle
$$

Combining Eqs. (31) and (32) together, we arrive at the following expression for the size distribution function $f(v, \Theta)$ evaluated at a given initial distribution $f(v, 0) \equiv f_{0}(v)$ :

$$
f(v, \Theta)=\mathcal{L}^{-1}\left[\frac{(1-\vartheta) \Xi_{0}(p, \Theta)}{1-\vartheta \Xi_{0}(p, \Theta)}\right], \quad \Xi_{0}(p, \Theta)=\int_{0}^{\infty} e^{-p v \exp (\beta \Theta)} f_{0}(v) d v,
$$

where $\mathcal{L}^{-1}[X]$ stands for the inverse Laplace transform of $X$, which in case of an arbitrary initial size distribution $f_{0}(v)$ has to be evaluated numerically. However, the explicit analytical expression can be provided for some physically relevant cases.

In the large size limit, $e^{-\beta \Theta} v / v_{0} \gg 1$, the frequency domain can be safely restricted to the $p$-values respecting strong inequality $p v_{0} e^{\beta \Theta} \ll 1$ allowing the approximate expansion, $e^{-p v \exp (\beta \Theta)} \approx 1-p v \exp (\beta \Theta)$, and leading to the following expression for $f(v, \Theta)$ :

$$
f(v, \Theta) \approx \frac{1}{v_{0}}\left[1+\frac{\kappa_{0} \Theta}{2 v_{0}}\right]^{-1} \exp \left[-\beta \Theta-\frac{e^{-\beta \Theta} v / v_{0}}{1+\kappa_{0} \Theta /\left(2 v_{0}\right)}\right], \text { at } \frac{e^{-\beta \Theta_{v}}}{v_{0}} \gg 1
$$

In the large size and long time limits, the last equation reduces to:

$$
f(v, \Theta) \approx \frac{2}{\kappa_{0} \Theta} \exp \left[-\beta \Theta-\frac{2 e^{-\beta \Theta} v}{\kappa_{0} \Theta}\right], \text { at } \frac{e^{-\beta \Theta} v}{v_{0}} \gg 1, \frac{\kappa_{0} \Theta}{2 v_{0}} \gg 1,
$$

showing that the distribution function no longer depends neither on the initial size distribution nor on the initial average volume $v_{0}$, as typically encountered in classical coagulation problems [Aldous (1999)]. Furthermore, in absence of the aggregate growth between coalescence events, $\beta=0, \Theta=\tau$, the equation (35) reduces to the well-known solution of the Smoluchowski equation with the constant kernel, which is conventionally written as follows [Aldous (1999)]: $\psi(v, \tau)=M_{0}(\tau) f(v, \tau) \approx\left[2 / \tau^{\prime}\right]^{2} \exp \left[-2 v / \tau^{\prime}\right]$, at $v / v_{0} \gg 1, \tau^{\prime} /\left(2 v_{0}\right) \gg 1$, with $\tau^{\prime}=$ $\kappa_{0} \tau$.

The asymptotic solutions [Eqs. (34) and (35)] typically provide valuable information on the size distribution shape in the broad volume interval predicting a continuous decrease of the distribution function $f(v, \Theta)$ with increasing dimensionless volume $v$. However, they lose the details of the size distribution shape at small volumes, such as an initial increase of $f(v, \Theta)$ with $v$. These details can be captured with the exact solution [Eq. (33)]. For the special case of the initial size distribution

$$
f_{0}(v)=\frac{1}{m !}\left(\frac{m+1}{v_{0}}\right)^{m+1} v^{m} e^{-\frac{(m+1) v}{v_{0}}}, m \in \mathbb{N}_{0}
$$


relevant for our experiments at appropriately chosen value of $m$, the distribution function [Eq. (33)] is explicitly expressed through $v, \Theta$, as follows:

$$
\begin{aligned}
& f(v, \Theta)=\frac{1-\vartheta}{v_{0} e^{\beta \Theta \vartheta}} \sum_{j=1}^{m+1}\left[\left(1+\frac{v_{0} e^{\beta \Theta}}{m+1} p_{j}\right) e^{p_{j} v}\right] \\
& 1-\vartheta+\sum_{j=1}^{m+1}\left[C_{m+1}^{j}\left(\frac{v_{0} e^{\beta \Theta}}{m+1}\right)^{j} p_{j}\right]=0
\end{aligned}
$$

where $p_{j}$ are $(m+1)$ complex roots of the polynomial equation (37b), evaluated analytically at $m \leq 3$ or numerically at $m \geq 4 ; C_{m+1}^{j}$ are binomial coefficients, and the coefficient $\vartheta$ is expressed through the reduced time $\Theta$ by the last expression in Eq. (31). Notice that Eq. (37) reduces to Eq. (34) for $m=0$, i.e. for the exponential initial distribution $f_{0}(v)=v_{0}^{-1} e^{-v / v_{0}}$.

Finally, note that the time $(\tau)$ dependence of the size distribution is obtained in parametric form, $[f(v, \Theta), \tau(\Theta)]$ using one of the equations (33), (34), (35) or (37a) for $f(v, \Theta)$ and Eq. (28) for $\tau(\Theta)$ by varying the parameter $\Theta$ in the range $\Theta \in\left[0, \Theta_{\text {max }}\right]$, with $\Theta_{\text {max }}$ given by the numerical solution of Eq. (29). The obtained theoretical size distribution will be compared to the experimental one in Sec. IV-E.

\section{Experiments}

\section{A. Magnetic colloid}

The magnetic colloid (ferrofluid) composed of maghemite nanoparticles dispersed in water has been synthesized using the co-precipitation method [Massart (1981)] and stabilized against irreversible aggregation by adsorption of trisodium citrate allowing electro-steric repulsion between nanoparticles at neutral $\mathrm{pH}$ and at initial relatively low ionic strength $(\sim 0.05 \mathrm{M})$ [Dubois et al. (1999); Roger et al. (2006)]. The parent ferrofluid was stable against colloidal aggregation for a few years. For experiments on phase separation, the parent ferrofluid was diluted in Milli-Q water with the addition of $0.35 \mathrm{~mol} / \mathrm{L}$ of sodium chloride $(\mathrm{NaCl})$ salt and an appropriate amount of the hydrochloric acid $(\mathrm{HCl})$ to reach the $\mathrm{pH} \approx 5.5$ and the nanoparticle volume fraction $\varphi_{0}=1.6 \times 10^{-3}(0.16 \mathrm{vol} . \%)$. The mixture was stirred for $1 \mathrm{~h}$ using an orbital shaker. The salt addition allowed decreasing of the characteristic thickness of the electric double layer (Debye screening length) on the nanoparticle surface, while the addition of the acid allowed some decrease of the surface electric charge thanks to partial deprotonation of the carboxylate groups of adsorbed citrate ions. This protocol allowed therefore partial screening of electrostatic interactions between nanoparticles promoting slight colloidal agglomeration necessary to induce reversible phase separation in the presence of magnetic fields.

The hydrodynamic size distribution and electrophoretic mobility (or zeta-potential) of nanoparticles in the diluted colloid were determined by dynamic light scattering (DLS) and laser Doppler velocimetry using Zeta Sizer Nano ZS (Malvern Instruments, UK). The obtained intensity size distribution (i.e. the fraction of the intensity of the light scattered by particles of a given size range as a function of the particle size) is shown in Fig. 2 for different times $t$ elapsed since the dilution of the colloid, with $t=1 \mathrm{~h}$ corresponding to the moment immediately after the orbital shaking. For the sake of comparison, we add a size distribution curve measured 
on the dilute colloid $\left(\varphi_{0}=1.6 \times 10^{-3}\right)$ without salt/acid addition $(\mathrm{pH} \approx 7.6)$ - see dashed black curve in Fig. 2. This curve presents a single peak at $d_{H} \approx 21 \mathrm{~nm}$ that does not evolve with time showing perfect colloidal stability. The size distribution with salt/acid addition at $t=1 \mathrm{~h}$ is within the measurement error equivalent to the size distribution without salt/acid addition. However, at $t=2,3,4 \mathrm{~h}$, a secondary peak at $d_{H} \approx 120-140 \mathrm{~nm}$ appears and its height increases with time, while the height of the primary peak at $d_{H} \approx 20 \mathrm{~nm}$ decreases pointing out to progressive agglomeration of nanoparticles caused by the partial screening of electrostatic repulsion.

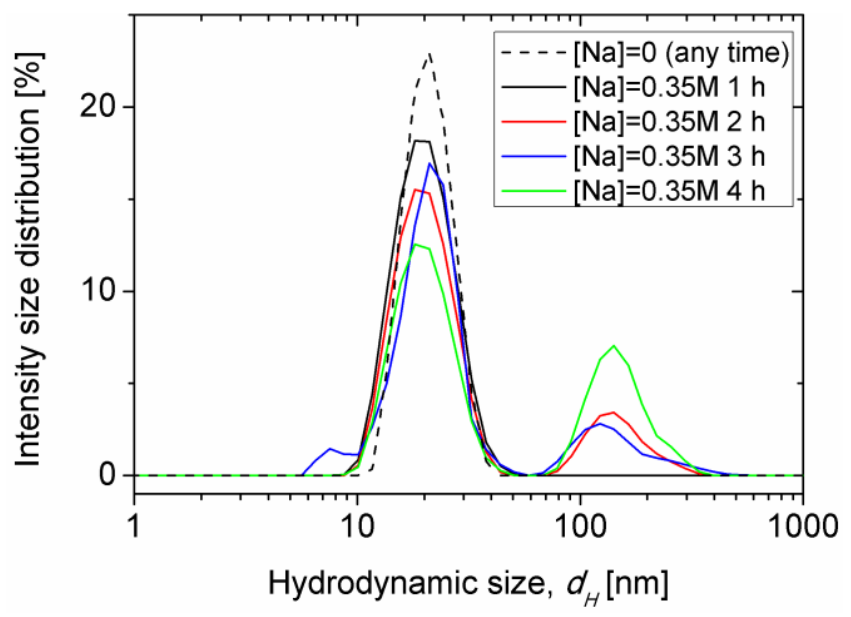

Fig. 2. The intensity distribution of hydrodynamic nanoparticle size measured by DLS

The electrophoretic mobility measurements are summarized in Table I and show that (a) in all the cases, the zeta potential remains negative pointing out to negative surface charge coming from carboxylate surface groups; (b) it is lower in absolute value for the colloid with salt/acid addition $(\sim-20 \mathrm{mV})$ than without addition $(\sim-50 \mathrm{mV})$ because of lower protonation degree of carboxylate groups at lower $\mathrm{pH}$; (c) it shows some increase (in absolute value) with the time likely because of long protonation equilibrium processes. To check possible electrostatic screening, the Debye screening length, $\kappa^{-1}$, is evaluated under assumption of symmetric 1-1 electrolyte with the total molar concentration of sodium ions equal to the sum of the concentration of added $\mathrm{NaCl}$ salt and initial concentration of sodium counterions measured by Inductive Coupled Plasma - Optical Emission Spectrometry (ICP-OES): $[\mathrm{Na}]_{\text {tot }}=[\mathrm{NaCl}]+[\mathrm{Na}]_{\text {in }}$ $=0.350+0.025=0.375(\mathrm{~mol} / \mathrm{L})$. Using the well-known expression for $\kappa^{-1}$ [see for instance van de Ven (1989)], we get $\kappa^{-1} \approx 0.5 \pm 0.1 \mathrm{~nm}$ with salt addition and $\kappa^{-1} \approx 2.0 \pm 0.1$ without salt/acid addition. We see a four-fold decrease of the Debye screening length with the salt addition which decreases colloidal stability against van der Waals dispersion forces. However, steric repulsion due to adsorbed citrate layers avoids fast irreversible aggregation. The samples prepared with salt/acid addition do not precipitate for at least a few months despite some agglomeration of nanoparticles revealed by DLS [Fig. 2]. The results of the above physicochemical characterizations are summarized in Table I for different elapsed times. 
Table I. Physicochemical characterizations of samples without $([\mathrm{Na}]=0)$ and with $([\mathrm{Na}]=0.35 \mathrm{M})$ salt/acid addition

\begin{tabular}{|l|l|l|l|l|}
\hline Sample & $\begin{array}{l}\text { Elapsed } \\
\text { Time, } \mathrm{h}\end{array}$ & $\mathrm{pH}$ & $\begin{array}{l}\text { Zeta } \\
\text { potential } \\
(\mathrm{mV})\end{array}$ & $\begin{array}{l}\text { Debye } \\
\text { length } \\
(\mathrm{nm})\end{array}$ \\
\hline$[\mathrm{Na}]=0$ & $0-1$ year & $7.60 \pm 0.05$ & $-49.5 \pm 3.0$ & $2.0 \pm 0.1$ \\
\hline \multirow{5}{*}[\mathrm{Na}]{$=0.35 \mathrm{M}$} & 1 & $5.50 \pm 0.05$ & $-18.8 \pm 2.2$ & $0.5 \pm 0.1$ \\
\cline { 2 - 5 } & 2 & $5.50 \pm 0.05$ & $-23.4 \pm 1.0$ & $0.5 \pm 0.1$ \\
\cline { 2 - 5 } & 3 & $5.55 \pm 0.05$ & $-25.3 \pm 1.7$ & $0.5 \pm 0.1$ \\
\cline { 2 - 5 } & 4 & $5.55 \pm 0.05$ & $-29.6 \pm 1.7$ & $0.5 \pm 0.1$ \\
\hline
\end{tabular}

Magnetization measurements have been performed on the parent ferrofluid at room temperature using a Vibrating Sample Magnetometry (VSM 4500 from EG\&G Princeton Applied Research, USA). These measurements allowed finding the magnetization saturation $M_{S} \approx 310 \pm 10 \mathrm{kA} / \mathrm{m}$ of nanoparticles relevant for maghemite composition with spin canting effects and the size distribution of their iron oxide cores fitted to log-normal distribution:

$$
f_{m}\left(d_{m}\right)=\frac{1}{\sqrt{2 \pi} \sigma_{m} d_{m}} \exp \left(-\frac{\left(\ln \left(\frac{d_{m}}{\left\langle d_{m}\right\rangle}\right)+\frac{\sigma_{m}^{2}}{2}\right)^{2}}{2 \sigma_{m}^{2}}\right)
$$

with the relative distribution width $\sigma_{m}=0.36$ and average diameter of the iron oxide cores (referred to as "magnetic" diameter) $\left\langle d_{m}\right\rangle=8.1 \mathrm{~nm}$. The "magnetic" nanoparticle size $\left\langle d_{m}\right\rangle=$ $8.1 \mathrm{~nm}$ is about 2.5 times less than the hydrodynamic size $d_{H} \approx 21 \mathrm{~nm}$ (at the primary peak) likely because of slight agglomeration even in absence of salt/acid addition.

The $j^{\text {th }}$ moment of the size distribution, intervening into evaluations of the internal aggregate volume fraction $\varphi^{\prime \prime}(j=3,6$, Eq. (A3)) and the critical nuclei volume $(j=9$, Eq. (6)) is given by

$$
\left\langle d_{m}^{j}\right\rangle=\left\langle d_{m}\right\rangle^{j} \exp \left(\frac{1}{2}(j-1) j \sigma_{m}^{2}\right)
$$

which gives $\left\langle d_{m}^{3}\right\rangle=(9.2 \mathrm{~nm})^{3},\left\langle d_{m}^{6}\right\rangle=(11.2 \mathrm{~nm})^{6},\left\langle d_{m}^{9}\right\rangle=(13.6 \mathrm{~nm})^{9}$.

\section{B. Experimental setup and measuring protocol}

The phase separation under rotating magnetic fields is experimentally observed inside a microfluidic channel placed in the center of two pairs of Helmholtz coils, as shown in Fig. $3 \mathrm{a}$. The two external coils of the $1^{\text {st }}$ pair are perpendicular to two internal coils of the $2^{\text {nd }}$ pair. The alternating (AC) sinusoidal voltage of the desired frequency in the range $v=5-25 \mathrm{~Hz}$ is generated by two audio channels of the motherboard of a personal computer controlled by a home-made MATLAB code. Both signals are amplified with the help of the Macro-Tech 5002 VZ sound amplifier (Crown Audio, USA) and are then injected into two coil pairs. The amplitude, amplification and phase lag of both voltage signals are carefully adjusted to get magnetic fields generated by each pair of coils having the same amplitude $H_{0}=9 \mathrm{kA} / \mathrm{m}$ and the phase lag of $\pi / 2$. This allows to produce homogeneous (on the length scale of the 
microfluidic channel) circularly polarized rotating magnetic field of an amplitude $H_{0}$ and an angular frequency $\omega=2 \pi \nu$.
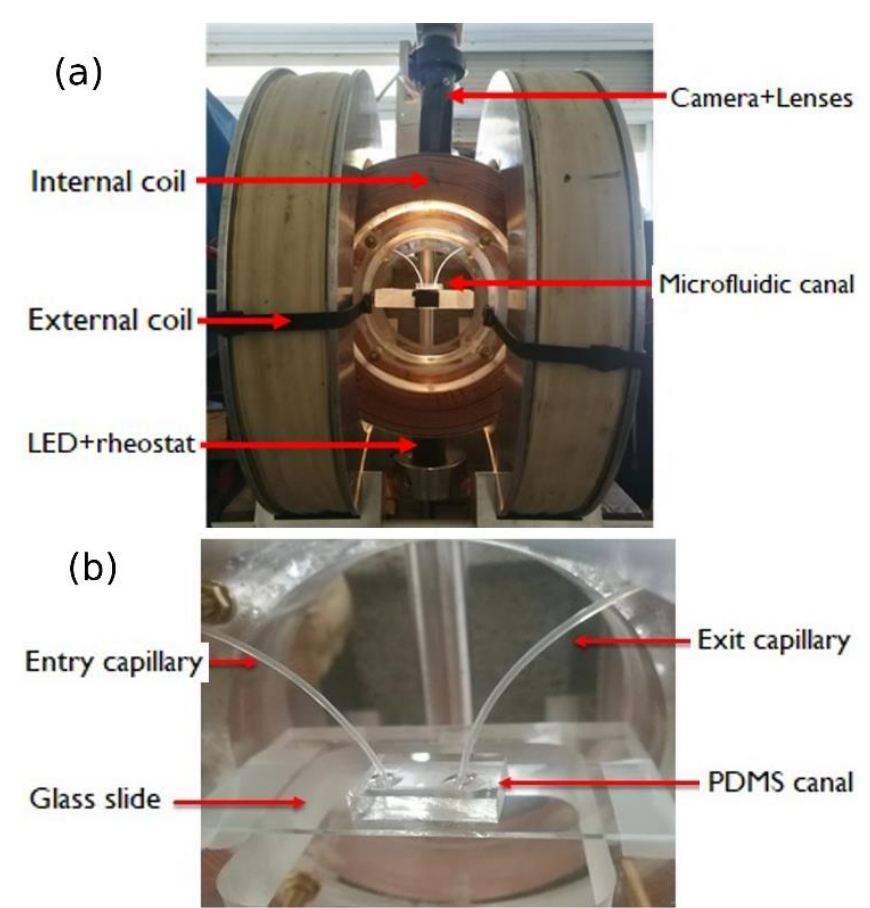

(c)

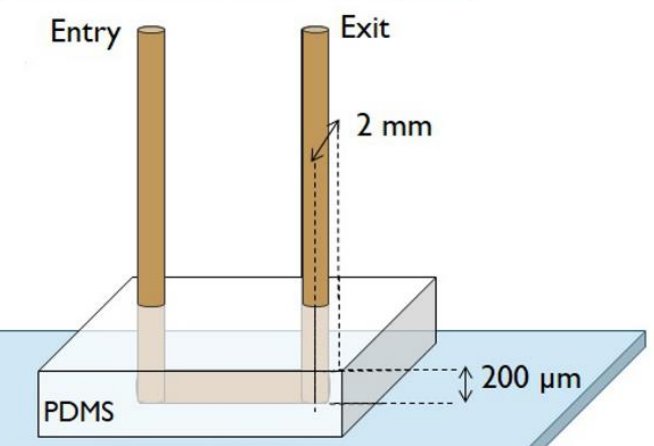

Glass Slide

Fig. 3. Experimental setup (a). A magnified view showing a microfluidic channel placed in the center of two pairs of coils is shown in (b). A sketch of the microfluidic channel is shown in (c)

A microfluidic channel was fabricated by gluing a poly dimethyl siloxane (PDMS) lid having a rectangular cavity to a microscope glass slide. The photographic and sketch views of the channel are shown in Figs. $3 b$ and $3 c$. The PDMS lid was fabricated by soft photolithography as described in detail in Ezzaier et al. (2018). The channel had a length of 1 $\mathrm{cm}$, a width of $2 \mathrm{~mm}$ and a thickness $h=200 \pm 5 \mu \mathrm{m}$. Flexible tubes of an internal/external diameter equal to $0.5 / 1 \mathrm{~mm}$ were introduced to both extremities of the channel to form the inlet and the outlet necessary for the filling with a magnetic colloid and washing with deionized water. All experiments were done with diluted colloid treated by salt/acid addition, having the particle volume fraction $\varphi_{0}=1.6 \times 10^{-3}$ and characterized in detail in Sec. III-A. After filling, the channel was placed in the middle between two coil pairs and the rotating magnetic field of the desired frequency was applied in the horizontal plane. The phase separation process was rapidly initiated by the magnetic field application and manifested through the appearance of 
needle-like nanoparticles aggregates synchronously rotating with the magnetic field, as supposed in the theoretical model [Sec. II-A] and sketched on Fig. 1a. The process was visualized with the help of InfiniTube ${ }^{\mathrm{TM}}$ Standard Video/Machine Vision Microscope (Infinity, USA) equipped with Infinity IF-4 objective and attached to a complementary metal oxide semiconductor (CMOS) detector camera PL-B742U (PixelLink, Ottawa, ON, Canada). The snapshots of the phase separating colloid were recorded each $0.25 \mathrm{~s}-$ a time interval small enough to follow the kinetics of aggregation after the very fast initial nucleation stage. Videos at a frame rate (in fps) equal to one-fourth of the given excitation frequency $v$ were also taken in some cases. The stack of the snapshots was analyzed by specially developed MATLAB code allowing determining the length and width distribution of the aggregates at each moment along with the integral characteristics, such as the number of aggregates per observation window, their average length, width, aspect ratio or volume, while the fraction of the suspension volume occupied by the aggregates was evaluated as $\Phi=n\langle V\rangle$. The image processing procedure can be summarized as follows. The first step was to get image stacks after correcting the illumination. The shading image was estimated as the temporal maximum of the time-lapse stack. Then, to correct illumination jittering, we extract a normalized absorbance stack $A(x, y, t)=[B-I(x, y, t)] / B$, where $I(x, y, t)$ is the image intensity after correction of inhomogeneity due to dust particles and illumination and $B$ is the most frequent illumination intensity. We then apply sequentially thresholding, morphological closing and removing small objects to get individual aggregates. For each aggregate, we get its absorbance and its length and width for a given moment. The histogram of the aggregate length distribution is finally constructed using a built-in MATLAB function. Notice that arrangement of all aggregates in the single horizontal plane significantly simplified the image processing. To check the correctness of the data processing by the MATLAB code, some results were checked with the Fiji software by "manual" measurements over a series of aggregates for each analyzed snapshot. All experiments where repeated 4 times and the integral characteristics were averaged over 5 measurements and the standard deviation was evaluated for each moment.

In addition to it, the amplitude of the threshold magnetic field $H_{0}$ and the threshold nanoparticle volume fraction $\varphi^{\prime}$ below which the field-induced agglomeration does not take place were measured by filling the microfluidic channel with the diluted colloid (treated with salt/acid addition) at different volume fractions $\varphi$, applying increasing values of the magnetic field intensity for $20 \mathrm{~min}$ and observing whether the needle-like aggregates of a minimal length about $1 \mu \mathrm{m}$ appear or not. The experimental dependencies $H_{0}\left(\varphi^{\prime}\right)$ were then analyzed for different field frequencies allowing determining the initial supersaturation $\Delta_{0}-$ the crucial parameter intervening into our theoretical model [Eqs. (19), (23)].

Since colloidal agglomeration (due to partial screening of electrostatic interactions between nanoparticles) could interfere with the field-induced aggregation, the sample history and duration of experiments are important issues for the present system. Each time, the experiments started one hour after the end of the orbital shaking of the sample. Once the experimental run lasted for $20 \mathrm{~min}$, the channel was washed and filled with a fresh sample, and a new experimental run was initiated. However, the whole experimental series lasted no longer than one hour. Thus, all the experiments fitted to the interval $2 \mathrm{~h} \leq t \leq 3 \mathrm{~h}$ of the time elapsed from the moment of the dilution of the parent ferrofluid (including orbital shaking stage lasting for 
$1 \mathrm{~h}$ - cf. Sec. III-A). According to DLS measurements [Fig. 2], the secondary size distribution peak is present, promoting efficient field-induced aggregation, but does not evolve significantly during this time interval. We expect therefore that timescale of colloidal agglomeration $(\sim \mathrm{a}$ few $\mathrm{h}$ ) is much longer than the timescale of the field-induced aggregation ( a few mins). This allows us to safely neglect the change of the suspension dispersion state during the field-induced phase separation.

\section{Results and discussion}

\section{A. Overview of observations results}

The optical images of the microstructure of the diluted magnetic colloid (after treatment with salt/acid) placed in a flat horizontal microfluidic channel of a thickness $h=200 \mu \mathrm{m}$, at a nanoparticle volume fraction $\varphi_{0}=1.6 \times 10^{-3}$, subjected to homogeneous magnetic field rotating in the horizontal plane and having the amplitude $H_{0}=9 \mathrm{kA} / \mathrm{m}$, are shown in Fig. 4 for four different frequencies (including $0 \mathrm{~Hz}$ - permanent field) and four different moments of elapsed time $t$. As seen from this figure, upon the magnetic field application, initially homogeneous magnetic colloid at $t=0$ (first row in Fig. 4 ) is subjected to a phase separation manifested through the appearance of needle-like micron-sized aggregates composed of a large number of nanoparticles. We have not managed to conduct reliable measurements in the permanent magnetic field $(v=0)$ because, the aggregates settled onto the bottom of the channel a few seconds after switching-on the field. Once settled, the aggregates were immobilized and stopped to grow. Despite settling, the aggregates kept their needle-like shape, as seen on the left column of Fig. 4.

In the rotating field (three right columns on Fig. 4), the aggregates rotate with the field in the clockwise direction without any visible settling. To check the rotational frequency of the aggregates, we have conducted a few supplementary short-time experiments using a high-speed camera. The rotational frequency of the aggregates coincides with the excitation frequency of the applied rotating magnetic field, as it was expected from Mason number evaluation by Eq. (1). At $M a<1$, the aggerate rotation is synchronous with the field, and in our experimental conditions we even get $M a \ll 1$. At relatively short elapsed times ( $t \gtrsim 5 \mathrm{~s}$ ), the major part of the aggregates migrates vertically withing the channel thickness to a single horizontal plane situated approximately in the middle between two horizontal channel walls. Such effect has already been observed by Stikuts et al. (2020) for magnetic colloid qualitatively similar to our one and has been interpreted in terms of hydrodynamic interactions with walls hindering gravitational settling.

Notice that in all the snapshots of Fig. 4, we observe micron-sized spherical particles whose location does not change with time. These are dust particles that probably come from the syringe joint and inevitably enter the microfluidic channel when it is reused several times for different experimental runs. Washing of the channel does not allow complete removal of these particles. Despite this drawback of our experimental system, we have never observed any distinguishable interaction between the dust particles deposited on the channel bottom and the magnetic aggregates rotating at the middle plane of the channel. Furthermore, the repeatability of experiments is confirmed for different states of the channel surface. 


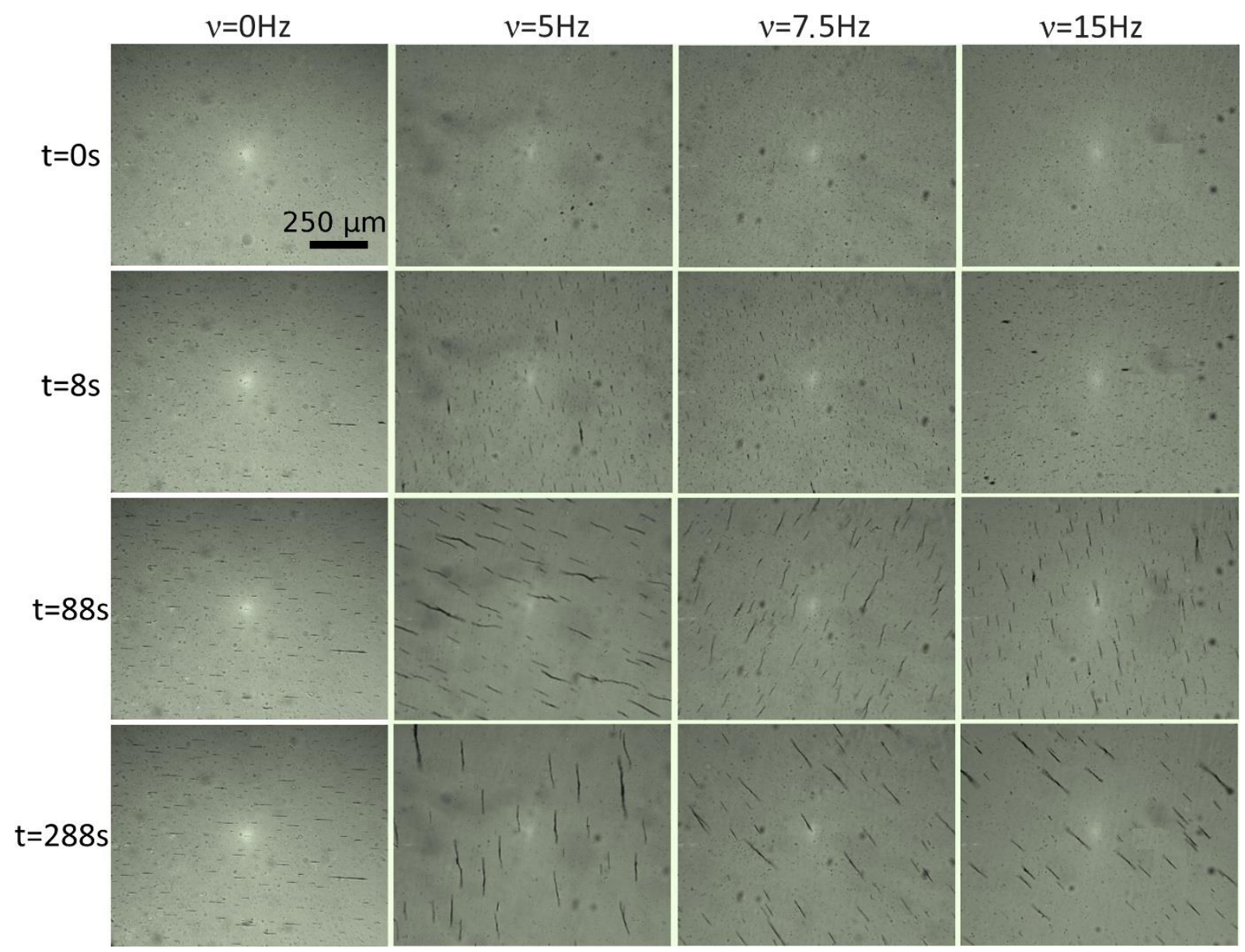

Fig. 4. Snapshots of field-induced aggregates under permanent and rotating magnetic fields. Rows correspond to the elapsed time (from the top to the bottom) $t=0,8,88$ and $288 \mathrm{~s}$. Columns correspond to the field frequencies $v=0,5,7.5$, and $15 \mathrm{~Hz}$. In the case of the permanent applied magnetic field (left column), the field is oriented horizontally. In the case of the rotating field (three right columns), the aggregates synchronously rotate with the field in the clockwise direction.

From the thermodynamic perspective, the aggregates are ascribed to the concentrated phase with the average particle volume fraction inside them evaluated to be $\varphi^{\prime \prime}=0.32 \pm 0.03$ [Appendix A], while the medium surrounding the aggregates is ascribed to the dilute phase with the average particle volume fraction $\varphi$ decreasing with time starting from its initial value $\varphi_{0}=$ $1.6 \times 10^{-3}$. These aggregates become visible at times as short as $t=8 \mathrm{~s}$ (second row of Fig. 4 ), and even at $t=1 \mathrm{~s}$ pointing out to fast kinetics of nucleation, as supposed in the model [Sec. II-A] and observed in video-a.avi in Supplemental Materials. The aggregate size progressively increases with time ( $3^{\text {rd }}$ and $4^{\text {th }}$ rows in Fig. 4 ) thanks to coalescence between them (which is explicitly observed in videos and confirmed by the visual decrease of the number of observed aggregates per observation window) combined with absorption of individual nanoparticles from dilute phase (which is implicitly observed as the aggregate growth between two coalescence events). At elapsed times $t \gtrsim 100 \mathrm{~s}$, the coalescence of two aggregates is often accompanied by their fragmentation into three or four shorter parts. This fragmentation seems to limit the aggregate average size which seems to no longer increase at $t \gtrsim 200 \mathrm{~s}$. Coalescence and a few fragmentation events are observed in video-b.mp4 in Supplemental materials. As opposed to the study of Stikuts et al. (2020), we do not observe the regular crystalline structure of 
monodisperse (in length) rotating aggregates at long times. The aggregate length in our case remains rather polydisperse (as analyzed in Sec. IV-E) which likely hinders any ordering. The observations are qualitatively similar for all studied field frequencies in the range $5 \leq v \leq$ $25 \mathrm{~Hz}$ (compare three right columns in Fig. 4). Fragmentation events are more frequent at higher frequency implying a decrease of the aggregate size with the frequency. Exact time dependence of the aggregate size will be analyzed in Sec. IV-D. Notice that blurry tips of the aggregates in two bottom rows of Fig. 4 are the result of relatively long exposure time of the low-speed CMOS camera. If the field is rapidly switched off, the aggregates stop spinning and we detect a sharp shape of their tips before the aggregates start to dissolve in the dilute phase under Brownian diffusion of nanoparticles.

Based on these primary observations, we will now study in detail the threshold of fieldinduced separation through the $H_{0}-\varphi$ phase diagram [Sec. IV-B], the minimal and maximal average size of the aggregates [Sec. IV-C], the integral characteristics of the phase separation [Sec. IV-D], and the aggregate size distribution [Sec. IV-E]; most of these experimental characteristics will be compared to predictions of our model.

\section{B. Phase diagram and initial supersaturation}

The amplitude of the threshold magnetic field $H_{0}$ of the phase separation was measured as a function of nanoparticle volume fraction $\varphi^{\prime}$ at different frequencies of the magnetic field, including $v=0$ (permanent field). Notice that gravitational settling in the permanent field is slow enough for small initial aggregates and does not hinder determination of the aggregation threshold. It has been found that within the experimental errors, the field frequency does not affect the $H_{0}\left(\varphi^{\prime}\right)$-dependency in the range $0 \leq v \leq 25 \mathrm{~Hz}$. Thus, the experimental $H_{0}\left(\varphi^{\prime}\right)$ dependency is plotted in Fig. 5 for the permanent magnetic field only. The error bars correspond to standard deviation determined from a series of three equivalent measurements. The $H_{0}\left(\varphi^{\prime}\right)$ dependency follows common trends typically observed for permanent fields [Buyevich and Ivanov (1992), Ezzaier et al. (2017)].

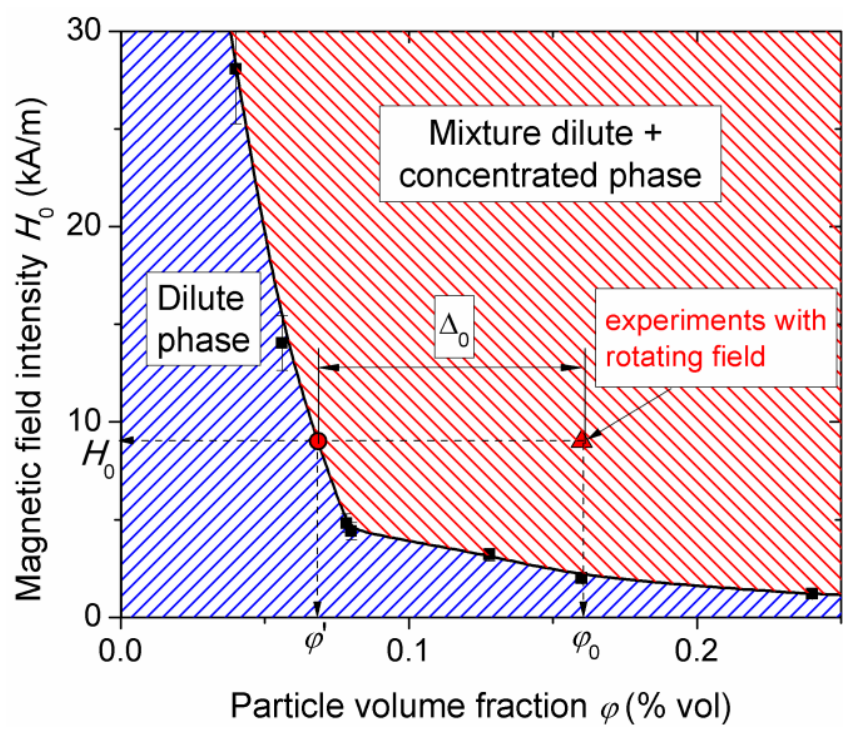

Fig. 5. $H_{0}-\varphi$ phase diagram of the diluted magnetic colloid treated by salt/acid addition in the permanent magnetic field. The phase diagram appears to be the same (within experimental error bars) in the rotating field in the frequency range $5 \leq v \leq 25 \mathrm{~Hz}$. 
First, the phase separation threshold is shifted to lower magnetic fields with increasing volume fraction $\varphi^{\prime}$. This is understood in terms of increasing dipolar interactions between nanoparticles, as the average distance between them decreases with increasing volume fraction. Second, the $H_{0}\left(\varphi^{\prime}\right)$-curve separates the $H_{0}-\varphi$ space into two regions, as follows: (a) dilute phase region on the left represented by a blue hatch on Fig. 5, where the phase separation cannot be induced by the applied field, and (b) the mixture of the dilute and concentrated phases on the right represented by a red hatch with the phase separation manifested through the appearance of needle-like aggregates (concentrated phase) surrounded by a suspension of individual non-aggregated nanoparticles (dilute phase). In this context, the $H_{0}-\varphi$ space can be considered as a phase diagram and $H_{0}\left(\varphi^{\prime}\right)$-curve - as the left binodal curve since the considered phase separation is nothing but the binodal decomposition of the magnetic colloid under the applied field. The second binodal curve $H_{0}\left(\varphi^{\prime \prime}\right)$ marking the transition between the bulk concentrated phase and the mixture of both phases is not easily accessible in experiments and thus not plotted in Fig. 5. Tedious experiments on hydrodynamic mobility of aggregates under magnetic field gradient allowed the determination of the volume fraction $\varphi^{\prime \prime}=0.32 \pm$ 0.03 in the concentrated phase for the single value of the magnetic field amplitude $H_{0}=$ $9 \mathrm{kA} / \mathrm{m}$ [Appendix A]. Third, the phase diagram allows experimental evaluation of the suspension initial supersaturation $\Delta_{0}$ as the difference between the nanoparticle volume fraction $\varphi_{0}=1.6 \times 10^{-3}$ in the suspension and the volume fraction $\varphi^{\prime}$ at the aggregation threshold. For the magnetic field amplitude $H_{0}=9 \mathrm{kA} / \mathrm{m}$ used in all kinetic experiments, we find $\varphi^{\prime} \approx$ $7 \times 10^{-4}$ and $\Delta_{0}=\varphi_{0}-\varphi^{\prime} \approx 9 \times 10^{-4}$. The initial supersaturation is graphically represented in Fig. 5 by a horizontal segment relating the point $\left(\varphi^{\prime}, H_{0}\right)$ on the binodal curve with the point $\left(\varphi_{0}, H_{0}\right)$ representing the initial state of our suspension at the beginning of the phase separation. Recall that the binodal curve does not depend on the field frequency, therefore, the value $\Delta_{0} \approx$ $9 \times 10^{-4}$ found for the permanent field remains valid for rotating field in the frequency range $0 \leq v \leq 25 \mathrm{~Hz}$.

The independence of the phase separation threshold of the frequency likely indicates that the phase equilibrium on the surface of aggregates between the dilute (outside the aggregates) and the concentrated (inside the aggregates) phases is not altered by aggregate rotation, at least in the considered frequency range. At all experimental frequencies, the aggregates have a high enough aspect ratio, $r_{a}>5$ and very small phase lag, $\phi \sim 0.1^{\circ}$, between their main axis and the field direction, as evaluated by Eq. (1). Under such conditions, the demagnetizing effects (arising due to refraction of the field lines on the aggregate surface) can be safely neglected. Thus, the distribution of the instantaneous magnetic field on the surface of the aggregates is supposed to be unaltered by the aggregate rotation and cannot change the phase equilibrium. On the other hand, the aggregate rotation generates the hydrodynamic pressure, $p_{H}$, evaluated in Appendix C [Eq. C9]. It can in principle change the phase equilibrium on the aggregate surface if it becomes comparable with either osmotic pressure, $p_{o s m}=n^{\prime} k_{B} T$, of nanoparticles in the dilute phase (at their number density $n^{\prime}$ ) or with the capillary pressure $p_{\text {cap }} \approx \sigma_{0} / b$ due to the aggregate surface tension $\sigma_{0}$. The capillary pressure is found to dominate over the osmotic one, and the characteristic ratio of hydrodynamic-to-capillary pressures reads: 


$$
\frac{p_{H}}{p_{c a p}} \sim \frac{\eta_{0} \omega r_{a} b}{\sigma_{0}\left(\ln \left(2 r_{a}\right)-1 / 2\right)} \sim \frac{\eta \omega L_{\max }}{\sigma_{0}\left(2 \ln \left(2 r_{\max }\right)-1\right)}
$$

Taking $\eta_{0} \approx 10^{-3} \mathrm{~Pa} \times \mathrm{s}$ for water, $\sigma_{0} \approx 1.5 \times 10^{-4} \mathrm{~N} / \mathrm{m} \quad[$ Appendix $\mathrm{B}$ ] and experimental values of $L_{\max }$ and $r_{\max }$ for each angular frequency $\omega$, we evaluate $p_{H} / p_{c a p} \lesssim$ $7.5 \times 10^{-3}$ in the frequency range, $5 \leq v \leq 25 \mathrm{~Hz}$, with, of course $p_{H} / p_{\text {cap }}=0$ in the permanent field $(v=0)$. Thus, the hydrodynamic pressure is negligible with respect to the capillary one and is not expected to affect the phase equilibrium. Finally, the aggregate halfthickness $b$ is found to be nearly constant with the frequency, such that the capillary pressure is expected to be roughly independent of $\omega$ and likely cannot change phase equilibrium at different frequencies. In summary, we have eliminated possible effects of demagnetizing field, hydrodynamic and capillary pressures on the change of the phase separation threshold with the frequency $\omega$ of aggregate rotation, in agreement with experiments.

\section{Minimal and maximal aggregate size}

Above the phase separation threshold, once the magnetic field is applied, the micron-sized needle-like aggregates appear on a timescale of $\sim 1 \mathrm{~s}$, much shorter than the timescale of the whole phase separation process $\left(\sim 10^{2} \mathrm{~s}\right)$. This initial fast kinetics is attributed to fast nucleation stage that is not accessible in our experiments. One can consider that the aggregate growth/coalescence stages start with some initial (minimal) average aggregate size corresponding (at the first approximation) to the average size of critical nuclei, evaluated in Sec. II-B. Experimentally, the minimal aggregate size can be found from extrapolation of the experimental aggregate length versus time curves (as shown below on Fig. 7) to the ordinate axis. In experiments, we get $L_{\text {min }}=17 \pm 5 \mu \mathrm{m}$ - a value that compares relatively well with the theoretical evaluation by [Eqs. (6a), (6b)], $L_{\min }=L_{c} \approx 12 \mu \mathrm{m}$, obtained at the considered parameters $\varphi_{0}=1.6 \times 10^{-3}$ (initial nanoparticle volume fraction), $\Delta_{0}=9 \times 10^{-4}$ (initial supersaturation - cf. Sec. IV-B), $H_{0}=9 \mathrm{kA} / \mathrm{m}$ (magnetic field amplitude) and $\left\langle d_{m}^{9}\right\rangle=$ $(13.6 \mathrm{~nm})^{9}$ (the $9^{\text {th }}$ moment of the size distribution of individual nanoparticles - cf. Eq. (39)). Notice that the size $d_{m}$ of individual nanoparticles (revealed from magnetization measurements - Sec. III-A) seems to be more relevant for the evaluation of the work of nucleus formation (and consequently critical nuclei size) than the hydrodynamic size $d_{p}$ revealed by DLS. Within experimental errors, experimental values of $L_{\min }$ appear to be independent of the field frequency within the range $0 \leq v \leq 25 \mathrm{~Hz}$, in agreement with the fact that the phase equilibrium on the aggregate (nucleus) surface is almost non-affected by the aggregate rotation, as discussed in detail in Sec. IV-B. Notice that, as in the case of the phase separation threshold, gravitational settling in the permanent magnetic field $(v=0)$ is slow enough with respect to the nucleation stage and still allows experimental determination of the minimal aggregate size.

At long times $(t \gtrsim 200 \mathrm{~s}$ ), the aggregate size is limited by fragmentation events coming from hydrodynamic interactions between aggregates. The experimental dependency of the maximal average aggregate length $L_{\max }$ on the field frequency $v$ is shown in Fig. 6 for the considered parameters $\varphi_{0}=1.6 \times 10^{-3}$ and $H_{0}=9 \mathrm{kA} / \mathrm{m}$. The experimental data have been fitted by Eqs.(8), (9a) with the adjustable parameter value of the lateral gap between aggregates set to $h_{0}=60 \mathrm{~nm}$, thus being equal to about three nanoparticle hydrodynamic diameters 
$\left\langle d_{p}\right\rangle \approx 21 \mathrm{~nm}$ [Fig. 2], which is consistent with the proposed model [Sec. II-C]. The following values of other parameters were used in calculations: $\eta_{0} \approx 10^{-3} \mathrm{~Pa} \times \mathrm{s}, \quad b \approx 2.5 \mu \mathrm{m}$ [Appendix $\mathrm{B}$ ], $\chi \approx 22 \pm 5$ [Appendix A]. The result of the fit is presented by a solid curve in Fig. 6. Both the theory and the experiments show a relatively strong decrease in $L_{\max }$ with $v$ that is explained by the fact that two colliding aggregates can transiently coalesce and then split into several parts due to surface instabilities whose probability increases with the increasing relative speed of collision (cf. details of this possible scenario in Sec. II-C). This phenomenon is typically observed in experiments. Two long colliding aggregates seem to merge for a short time and fragment into three-to-four parts - see video-b.mp4 in Supplemental Materials. Short timescale dynamics of fragmentation is inaccessible for our slow-speed recording equipment and is out of the scope of our study. In fact, the most important parameter stemming from this dynamics is the maximal aggregate size. Our simple energy balance approach developed in Sec. II-C allows a semiquantitative agreement with experiments on the maximal aggregate length, as seen in Fig. 6, without the need for detailed study of fragmentation that can be envisaged in future. Quantitatively, the maximal average aggregate length varies in the range $30-80 \mu \mathrm{m}$ in the considered frequency range, while the aggregate thickness remains almost constant and approximately equal to $5 \mu \mathrm{m}$. The aggregate thickness will be discussed in more details in the next Sec. IV-D.

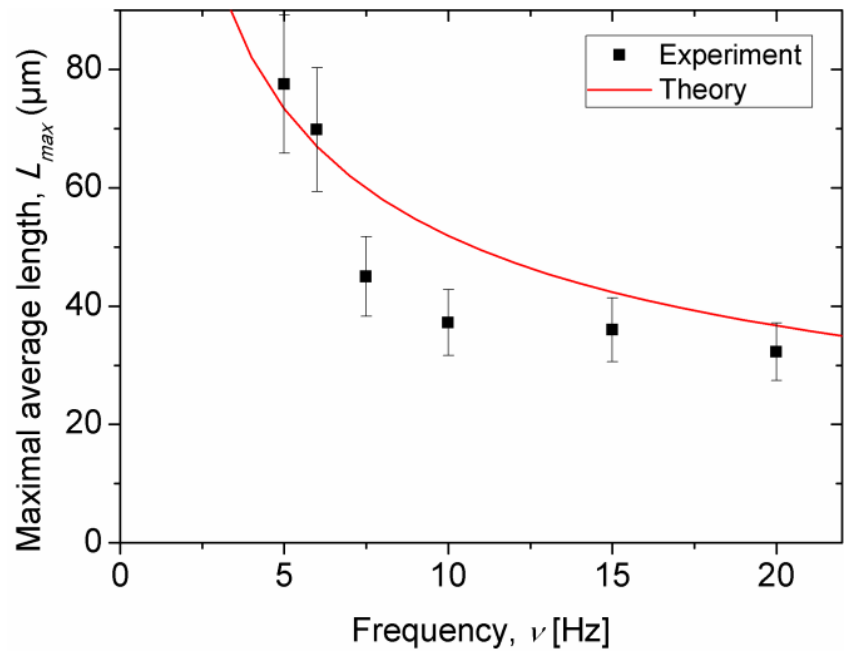

Fig. 6. The experimental and theoretical dependency of the maximal average aggregate length on the field frequency. The value of the adjustable parameter for the theoretical dependency [Eqs. (8), (9a)] is set to $h_{0}=$ $60 \mathrm{~nm}$.

Finally, a few supplementary measurements at different channel thicknesses show that the aggregate average maximal length [Fig. 6] and the phase diagram [Fig. 5] remain independent of the channel thickness in the studied range $50 \leq h \leq 200 \mu \mathrm{m}$.

\section{Integral characteristics}

Once the lower and upper limits for the aggregate size are found, we can inspect in detail the size evolution with time. The experimental dependencies of the average aggregate length $\langle L\rangle$ on elapsed time $t$ are shown in Fig. 7a for the field frequency $v=5 \mathrm{~Hz}$ and five different experimental runs. The inset presents the data at increased time scale. This graph shows a relatively large dispersion of data between different experiments at identical parameters, which 
is probably related to limited colloidal stability of the diluted magnetic colloid after salt/acid treatment and surface impurities of the microfluidic channel caused by imperfect washing of the channel between different runs. Despite such imperfect reproducibility, we have secured our data by error bars arising from standard deviation on five experimental sets for each frequency.

The experimental and theoretical $\langle L\rangle(t)$ dependencies obtained as an average on five identical measurements are shown in Fig. $7 \mathrm{~b}$ for $\varphi_{0}=1.6 \times 10^{-3}$ and $H_{0}=9 \mathrm{kA} / \mathrm{m}$ and at different magnetic field frequencies $v$. Notice that in the case of the permanent magnetic field $(v=0)$, the gravitational settling of aggregates does not allow obtaining reliable data at times $t \gtrsim 60 \mathrm{~s}$ because the settled aggregates are immobilized at the channel bottom and their further growth seems to be stopped after settling. For this reason, the average aggregate length in the permanent field (crossed black triangles on Fig. 7b) appears to be smaller than that in the rotating field at $v=5 \mathrm{~Hz}$. We would expect an opposite tendency if the gravitational settling were absent in the permanent field. Thus, the data of Fig. $7 \mathrm{~b}$ at $v=0$ should be handled with care. The theoretical curves at $v=10,15$ and $20 \mathrm{~Hz}$ are close to each other and only the theoretical curve for $v=15 \mathrm{~Hz}$ is shown in Fig. $7 \mathrm{~b}$.

The theoretical dependencies (solid lines) are calculated for the rotating field in a parametric form by varying the $\Theta$ parameter, respecting the following steps: (a) the average dimensionless volume $\langle v\rangle(\Theta)$ and time $\tau(\Theta)$ are evaluated using Eqs. (27c), (28); (b) the dimensional time is evaluated as $t=t_{d} \tau$ with the diffusion timescale $t_{d}$ given by Eq. (23); (c) at approximately constant aggregate semi-thickness $b \approx$ const, the equality $\langle v\rangle=\langle V\rangle / V_{\text {max }} \approx$ $\langle L\rangle / L_{\max }$ allows us to evaluate the average aggregate length as $\langle L\rangle \approx L_{\max }\langle v\rangle$. The following values of the physical parameters intervening into different calculation steps were used: the thickness of the microfluidic channel $h=200 \mu \mathrm{m}$; the average nanoparticle diameter $d_{p}=$ $21 \mathrm{~nm}$ was taken from the primary peak of the hydrodynamic size distribution [Fig. 2] - a value more relevant for the evaluation of nanoparticle Brownian diffusivity and diffusion timescale [Eq. (23)] than the size of metal oxide cores of individual nanoparticles; the initial supersaturation $\Delta_{0}=9 \times 10^{-4}$ found from the experimental phase diagram [Sec. IV-B, Fig. 5]; initial volume fraction of aggregates in the colloid $\Phi_{0}=3 \times 10^{-5}$ found from experimental snapshots at elapsed time $t=1 \mathrm{~s}$ by image processing; the aggregate half-thickness $b \approx$ $2.5 \mu \mathrm{m}$ [Appendix B], magnetic susceptibility $\chi \approx 22 \pm 5$ and internal volume fraction $\varphi^{\prime \prime}=$ $0.32 \pm 0.03$ [Appendix A]; the minimal average aggregate length $L_{\text {min }}=L_{c} \approx 12 \mathrm{~nm}$ corresponding to the critical nuclei size evaluated in Sec. IV-C; the maximal average aggregate length $L_{\max }$ can in principle be evaluated theoretically by Eqs. (8), (9a) but to provide better correspondence with experiments, we take experimental values of $L_{\max }$, shown by points in Fig. 6; finally, the initial average dimensional volume is evaluated as $v_{0}=V_{c} / V_{\text {max }} \approx$ $L_{c} / L_{\max }$. 

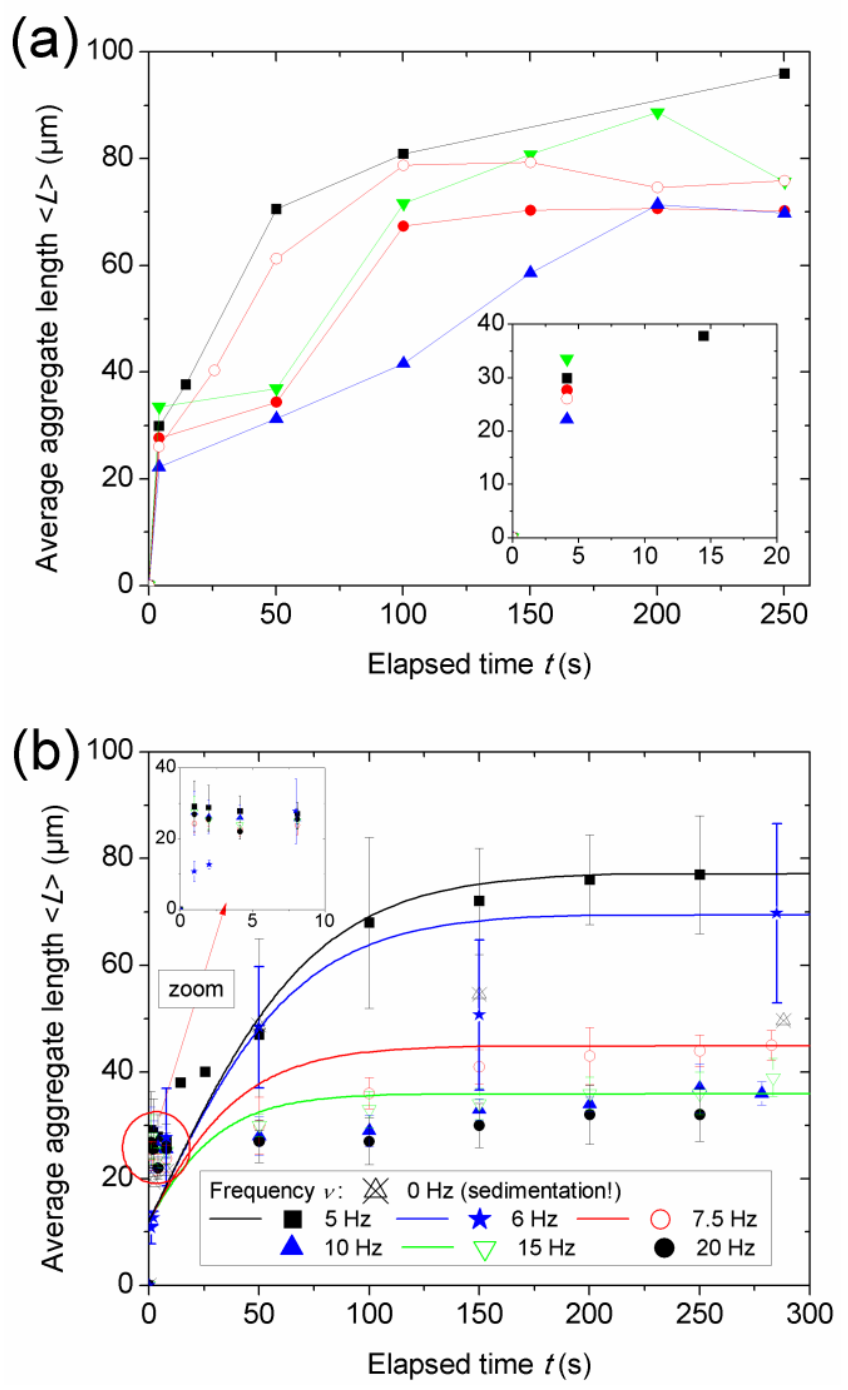

Fig. 7. The experimental time dependencies of the average aggregate length for five independent measurements at the frequency $v=5 \mathrm{~Hz}$ (a). The lines in (a) are guides for the eye. The experimental and theoretical time dependencies of the average aggregate length (b), each experimental curve being a medium of five independent measurements and error bars calculated as standard deviation. The points in (b) correspond to experiments and solid lines - to the theory. The insets in (a) and (b) present the data at increased time scale.

As is seen in Fig. 7b, the average aggregate length progressively increases with the elapsed time from the minimal value $L_{\text {min }}=17 \pm 5 \mu \mathrm{m}$ and achieves a plateau at long times $t \gtrsim 200 \mathrm{~s}$. The value $L_{\max }$ at the plateau decreases with increasing frequency in agreement with Fig. 6 . This plateau is ascribed to the equilibrium between the aggregate fragmentation rate and the effective aggregate growth rate including coalescence and absorption of nanoparticles by aggregates between coalescence events. This balance is believed to lead to saturation of the aggregate size. Our model seems to fit relatively well the experimental data, except for short times $t \lesssim 25 \mathrm{~s}$ where experiments predict a faster increase of the aggregate size with time, as shown on the inset of Fig. 7b. This disagreement likely comes from approximations on the aggregate growth rate $\dot{V} \propto(1-\langle v\rangle)$ [Eq. (19)] and coalescence constant $K=K_{0}(1-\langle v\rangle)$ [Eq. (21)], introduced to cancel the aggregate growth at $\langle v\rangle \rightarrow 1$ or $\langle V\rangle \rightarrow V_{\max }$ without introducing fragmentation terms into the population balance equation [Eq. (2)]. In reality, this approximation is expected to underestimate the quantities $\dot{V}$ and $K$ at short times (well before 
the fragmentation starts), thus, predicting slower aggregate growth than that observed in experiments. Despite this disagreement at short times our model for integral characteristics has the advantage to be free of any adjustable parameter; all physical quantities have been evaluated either experimentally or theoretically as specified above.

Recall that our model involves both aggregate growth (by convective diffusion of nanoparticles) and aggregate coalescence mechanisms through the population balance equation [Eqs. (2) and (25)]. The key parameter describing the transition between both mechanisms is the ratio of diffusion-to coalescence timescales, $\kappa_{0}=t_{d} / t_{c}$, whose value varies in the range 1.3-3.5 within the experimental frequency range $5 \leq v \leq 25 \mathrm{~Hz}$. This indicates that both mechanisms appear at quite similar timescales, $t_{d} \sim t_{c} \sim 15-60 \mathrm{~s}$ in rotating magnetic field, with coalescence being even somewhat faster than aggregate growth, as opposed to what is observed in the permanent magnetic field [Ezzaier et al. (2017)]. Faster coalescence in the rotating field could come from larger average dipolar interaction between rotating aggregates, as compared to non-rotating aggregates in the permanent magnetic field. The analysis shows, that in point dipole approach, the ratio of the coalescence constants in rotating and permanent fields scales as $K_{0}^{r o t} / K_{0}^{p e r m} \sim(h / b)^{5 / 2} r_{a}^{-3 / 2} \sim 10^{3}$ in agreement with faster coalescence in rotating fields.

Another important parameter is the constant $\beta=1 /(1+\alpha)=1 /\left(1+8\left(\ln \left(2 r_{\text {max }}\right)-\right.\right.$ $\left.1 / 2)^{1 / 3} P e_{\max }^{-1 / 3} / k\right)$ [cf. Eq. (17b)] governing the physics of the aggregate growth, with $k \approx$ 1.931. As follows from the analysis of Sec. II-D, the values $\beta \approx 1$ correspond to dominant diffusive transport of nanoparticles towards the cavity swept by the aggregate rotation, for which the aggregate growth timescale is given by the diffusion time $t_{d}$ [Eq. (23)]. On the opposite limit, $\beta \approx 1 / \alpha=k P e_{\max }^{1 / 3} /\left(8\left(\ln \left(2 r_{\max }\right)-1 / 2\right)^{1 / 3}\right) \ll 1$ with still high Peclet numbers $P e_{\text {max }} \gg 1$, the particle transport towards the aggregates is dominated by the convective diffusion through a thin diffusive boundary layer, and the characteristic aggregate growth timescale becomes $t_{c o n v}=t_{d} / \beta \approx 8\left(\ln \left(2 r_{\max }\right)-1 / 2\right)^{1 / 3} P e_{\max }^{-1 / 3} t_{d} / k$ and decreases with the field frequency as $t_{c o n v} \propto \omega^{-1 / 3}$. In our experimental case, the constant $\beta$ varies in a narrow range $\beta=0.53-0.62$ at the frequencies $5 \leq v \leq 25 \mathrm{~Hz}$ indicating that both mechanisms of the aggregate growth are comparable and cannot be considered separately.

In what concerns the aggregate thickness, $2 b$, experiments show that, within the experimental errors, it depends on neither the aggregate volume $V$, nor aspect ratio $r_{a}$, nor magnetic field frequency and is equal to $2 b \approx 5 \mu \mathrm{m}$, in quantitative agreement with the evaluation conducted in Appendix B. This result is in stark disagreement with previous theoretical models [Zubarev and Ivanov (1997); Ezzaier et al. (2017)] predicting $2 b \propto V^{2 / 7} \propto$ $r_{a}^{2}$. The difference arises in the details of the energy minimization. In the present model, we take into account possible variation of the surface energy with the aggregate magnetization, which allows better agreement with experiments on the aggregate shape and shows that the aggregate thickness behavior varies from $2 b \approx$ const at $12 \chi \gg r_{a}^{2} \gg 1$ to the conventional behavior $2 b \propto V^{2 / 7} \propto r_{a}^{2}$ at $r_{a}^{2} \gg 12 \chi \gg 1$ [see Appendix B]. 
To further inspect predictions of our model, the experimental and theoretical time dependencies of ratios $\Phi(t) / \Phi_{0}$ and $n(t) / n_{0}$ of volume fractions and number densities of aggregates are shown in Fig. 8 for the magnetic field frequency $v=7.5 \mathrm{~Hz}$ and at $\varphi_{0}=$ $1.6 \times 10^{-3}$ and $H_{0}=9 \mathrm{kA} / \mathrm{m}$. These dependencies have a similar shape at other frequencies. The experimental dependencies were obtained by averaging over five identical measurements and the error bars correspond to the standard deviation evaluated on the set of five measurements. Theoretical dependencies were calculated in parametric form using Eqs. (27a), (27b) and (28), using the same set of physical parameters as for the evaluation of the aggregate average length. Both the experiments and the model show: (a) an increase of the volume fraction of aggregates $\Phi(t)$ with time corresponding to the aggregate growth by absorption of nanoparticles from the dilute phase; and (b) a decrease of the aggregate number density $n(t)$ with time pointing out to coalescence of aggregates. The variation of both magnitudes occurs on the same timescale, $t \sim 60 \mathrm{~s}$, followed by saturation at longer times. This saturation corresponds to the limitation of the aggregate size to its maximal average value through fragmentation events. Our model predicts the following expressions for the plateau values of $n(t \rightarrow \infty) / n_{0}=n_{\min } / n_{0}$ and $\Phi(t \rightarrow \infty) / \Phi_{0}=\Phi_{\max } / \Phi_{0}$ obtained from Eqs. (27a), (27b):

$$
\frac{n_{\min }}{n_{0}}=\left[1+\frac{\kappa_{0} \Theta_{\max }}{2 v_{0}}\right]^{-1}, \quad \frac{\Phi_{\max }}{\Phi_{0}}=e^{\beta \Theta_{\max }},
$$

where $\Theta_{\max }$ is found from the solution of the transcendental equation (29).

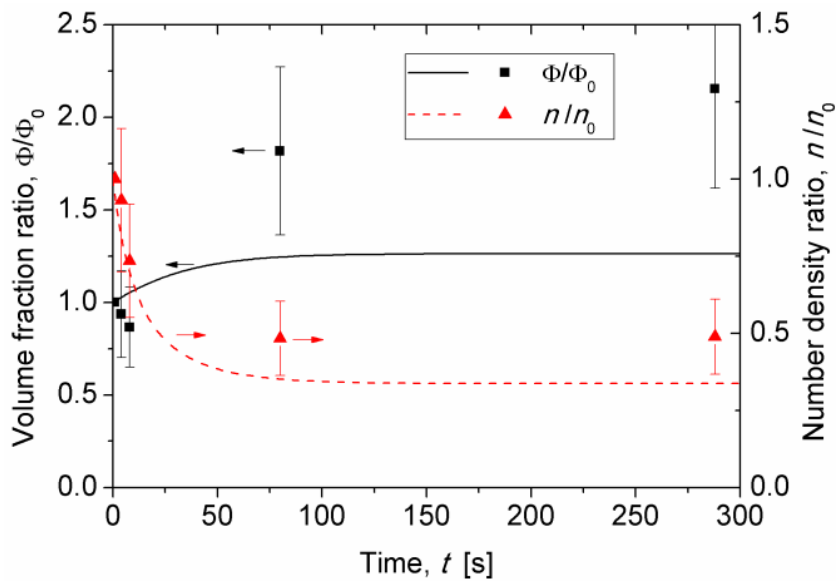

Fig. 8. Theoretical and experimental time dependencies of the ratios $\Phi(t) / \Phi_{0}$ (left ordinate axis) and $n(t) / n_{0}$ (right ordinate axis) of volume fractions and number densities of aggregates at the field frequency $v=7.5 \mathrm{~Hz}$. Symbols correspond to experiments and solid lines - to the theory.

Quantitatively, our model predicts a more important variation of the aggregate number density $n(t)$ and a weaker variation of the volume fraction of aggregates $\Phi(t)$ than those observed in experiments, even though large experimental errors could partially cover this difference. The discrepancy likely comes from the approximations $\dot{V} \propto(1-\langle v\rangle)$ [Eq. (19)] and $K=K_{0}(1-$ $\langle v\rangle)$ [Eq. (21)] which probably underpredicts the plateau values of $\Phi_{\max }$ and $n_{\min }$ given by Eq. (41). It worth noticing that experimental $\left(\Phi_{\max } \approx 6.6 \times 10^{-5}\right)$ and theoretical $\left(\Phi_{\max } \approx\right.$ $3.8 \times 10^{-5}$ ) values of the maximal concentration are two orders of magnitude smaller than the value $\Phi_{\max }=\Delta_{0} / \varphi^{\prime \prime} \approx 3 \times 10^{-3}$ corresponding to the thermodynamic equilibrium between 
dilute and concentrated phases. This conclusion holds within the whole experimental frequency range $5 \leq v \leq 25 \mathrm{~Hz}$ and clearly shows that the phase separation stops well before achieving thermodynamic equilibrium because of hydrodynamics interactions leading to fragmentation of aggregates. Our model qualitatively captures this physics and predicts a correct order of magnitude of the plateau values $\Phi_{\max } / \Phi_{0}$ and $n_{\min } / n_{0}$.

\section{E. Size distribution}

Once the integral characteristics of the phase separation are analyzed, we can proceed with the aggregate size distribution. Experimental and theoretical distribution functions $f(v)$ of the dimensionless volume $v$ are plotted in Figs. 9a-d for different moments of time $t$, at the field frequency $v=5 \mathrm{~Hz}$ and at $\varphi_{0}=1.6 \times 10^{-3}$ and $H_{0}=9 \mathrm{kA} / \mathrm{m}$. These dependencies have a similar shape for other frequencies. Experimental dependencies were constructed by the image processing procedure [Sec. III-B] and the data on Fig. 9 present the average over five independent measurements with the error bars standing for standard deviation over the set of these five measurements. Notice that the measured length distribution $f\left(L / L_{\max }\right)$ is equivalent to the distribution $f(v)$ over dimensionless volumes presented on Fig. 9, provided that $v=$ $V / V_{\text {max }} \approx L / L_{\max }$ at nearly constant aggregate thickness $2 b \approx$ const [see Sec. IV-D, Appendix B].

Experiments reveal a relatively narrow but finite-width size distribution of aggregates at the elapsed time $t=1 \mathrm{~s}$ [Fig. 9a]. This aggregate size distribution is characterized by a similar dimensionless average volume, $\langle v\rangle \approx v_{0}$ as the one of the critical nuclei $v_{0} \approx L_{\min } / L_{\max } \approx$ 0.16 with the critical nuclei length $L_{\min }=L_{c} \approx 12 \mu \mathrm{m}$ evaluated in Sec. IV-C. This allows us to suppose that the aggregate size distribution at short timescales, $t \sim 1 \mathrm{~s}$, corresponds to the size distribution of the critical nuclei at the end of the fast heterogeneous nucleation stage. This initial size distribution likely arises as a result of a polydispersity of the condensation centers (primary agglomerates ascribed to the secondary peak of the hydrodynamic size distribution [Fig. 2]) combined with complex kinetics of nucleation. As already mentioned, we do not have experimental access to the fast nucleation stage and its precise modelling is out of scope of the present paper. Thus, the initial size distribution of aggregates at short times, $t=1 \mathrm{~s}$, is fitted to the empirical function $f_{0}(v)$ [Eq. (36)] with $v_{0} \approx 0.16$ and the parameter $m$ describing the distribution width is set to $m=6$. The fit is presented by a solid curve in Fig. 9a. This empirical initial size distribution $f_{0}(v)$ is then used to calculate the theoretical aggregate size distribution $f(v)$ at different moments of time $t>1 \mathrm{~s}$ using Eq. (37). The theoretical size distributions at $t=8,80$ and $288 \mathrm{~s}$ are presented by solid curves in Figs. $9 \mathrm{~b}, \mathrm{c}$ and d. Evaluations of $f(v)$ by a long-time/large volume limit [Eq. (35)] are added as dashed curves for the sake of comparison. All the calculations have been done for the same set of physical parameters used for evaluations of the integral characteristics [Sec. IV-D]. To get the distribution function at a fixed elapsed time, $t=t_{d} \tau$, we first evaluate the reduced time $\Theta$ corresponding to the desired value of $t$ and then evaluate $f(v, \Theta)$ using Eq. (35) or Eq. (37). 

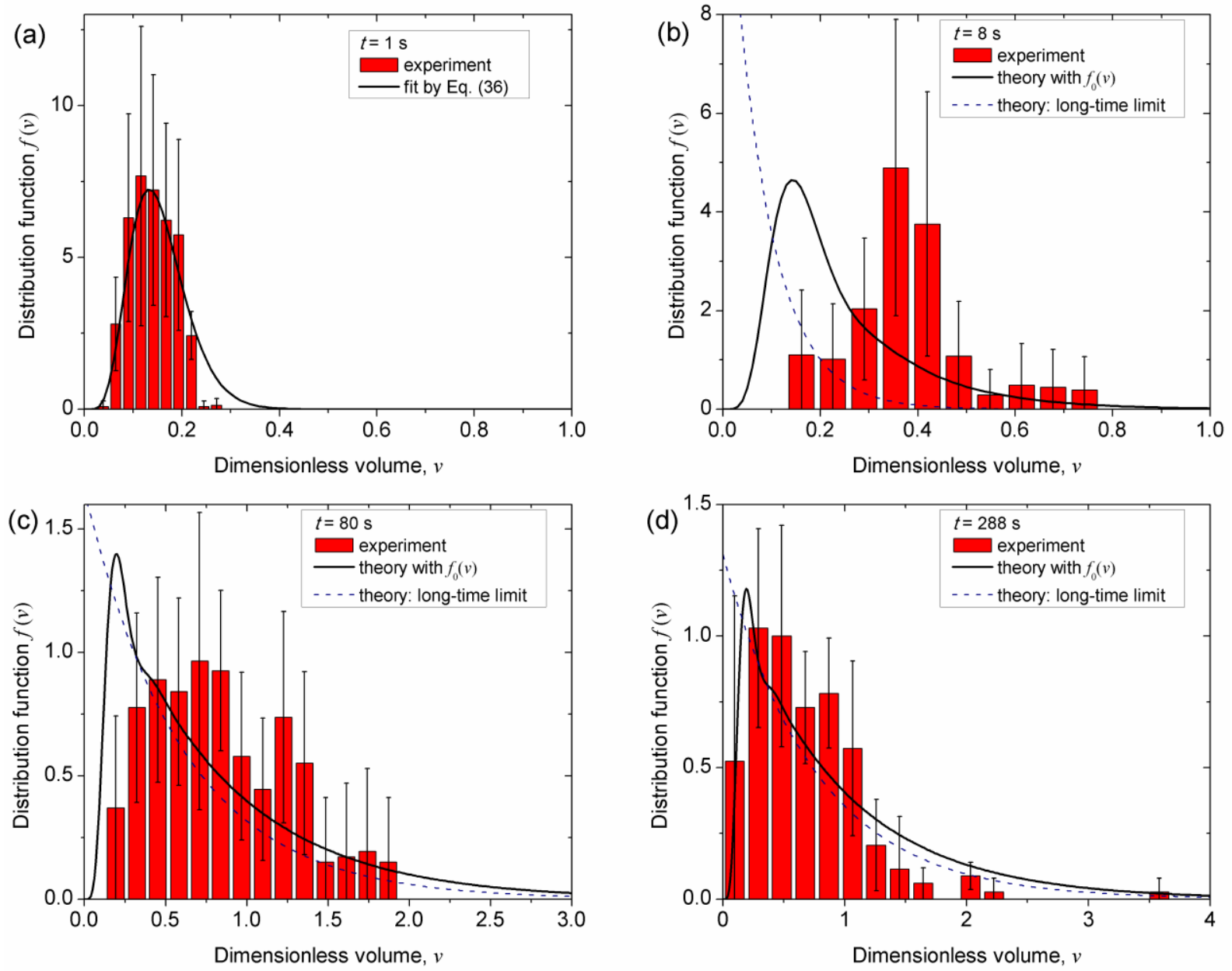

Fig. 9. Experimental and theoretical distribution of the aggregate dimensionless volumes for the frequency $v=$

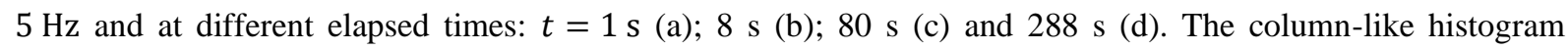
corresponds to experimental data obtained by averaging over five individual measurements. The solid curve in (a) corresponds to the fit of the experimental size distribution $f_{0}(v)$ by Eq. (36) at short time $t=1 \mathrm{~s}$. The curves in (b), (c), (d) stand for predictions of the model taking into account the initial size distribution $f_{0}(v)$ [solid curves, Eq. (37)] and the model in a long time/large volume limit [dashed curves, Eq. (35)].

Figures 9b-d show that the experimental size distribution spreads over the volume axis during the time. Such a spread is qualitatively reproduced by our model and is mostly related to the coalescence of unequal-sized aggregates broadening the size population, as typically observed in classical Smoluchowski limit [Aldous (1999)]. However, the model does not capture the significant shift of the distribution peak to larger volumes, especially at $t=8 \mathrm{~s}$, even though the average dimensionless volumes $\langle v\rangle$ given by experimental and theoretical distributions are quite similar, as inferred from Fig. 7b. Such a shift is typically reproduced by the model considering only the aggregate growth mechanism ignoring the coalescence [Kuzhir et al. (2020)]. This allows us to suppose that the relative contribution of coalescence in the present model is overestimated especially at short times, possibly due to approximations $\dot{V} \propto$ $(1-\langle v\rangle)$ [Eq. (19)] and $K=K_{0}(1-\langle v\rangle)$ [Eq. (21)]. However, at the present set of physical parameters, the coalescence-free approximation gives an extremely sharp and narrow size distribution not reproducing at all the present experimental data. It is worth noticing that the long time/large volume limit [Eq. (35)], conventionally used in coagulation problems [Levich 
(1962); Aldous (1999)], provides predictions close to the exact calculation by Eq. (37) at times $t \geq 80 \mathrm{~s}$ [Figs. 9c, d], except for small dimensionless volumes, $v \lesssim 0.5$, being unable to reproduce initial increase of the distribution function. Recall that, in a long time/large volume limit, the aggregate size distribution no longer depends on neither the shape nor the average volume $v_{0}$ of the initial size distribution, which is a pertinent feature of the considered coagulation-condensation process. However, in our particular case, the long time limit is strictly speaking not achieved even at infinite times. This is because this limit has been formulated for the compressed dimensionless timescale, $\Theta$, achieving finite values $\Theta_{\max } \approx 0.3-0.8$ at $t \rightarrow$ $\infty$, such that $\kappa_{0} \Theta /\left(2 v_{0}\right) \approx 1.4-3.4$ violating the "long time" condition, $\kappa_{0} \Theta /\left(2 v_{0}\right) \gg 1$. This is the reason for which the exact agreement between the exact calculations [Eq. (37)] and the long time limit [Eq. (35)] is never observed at infinite times, even though the difference is relatively small. Physically, the compressed timescale $\Theta$ allows a limitation of the size of rotating aggregates at $t \rightarrow \infty$ (introduced through approximations $\dot{V} \propto(1-\langle v\rangle)$ and $K=$ $K_{0}(1-\langle v\rangle)$ ), unlike the case of classical irreversible coagulation [Levich (1962); Aldous (1999)] or condensation-driven coagulation [Hassan and Hassan (2008)] when the aggregates grow indefinitely within the limits dictated by material balance. In our case, the final aggregate size distribution at $t \rightarrow \infty$ can be evaluated by Eq. (35) or Eq. (37) upon setting $\Theta=\Theta_{\max }$, with $\Theta_{\max }$ found from the solution of the transcendental equation (29).

\section{Concluding remarks}

This paper reports experimental and theoretical studies of the phase separation of a colloidal suspension of magnetic nanoparticles under circularly polarized rotating magnetic field in the frequency range $5 \leq v \leq 25 \mathrm{~Hz}$ allowing synchronous rotation of field-induced aggregates. In experiments, we use dilute aqueous suspensions of citrate-coated iron oxide nanoparticles slightly destabilized by increasing ionic strength and decreasing the suspension $\mathrm{pH}$, which allows the appearance of primary agglomerates of typical hydrodynamic size $\sim 150 \mathrm{~nm}$. They are expected to play the role of condensation centers for fast heterogeneous nucleation of nanoparticles in the presence of external magnetic fields. Beyond the first nucleation stage, occurring at the timescale less than $1 \mathrm{~s}$, the experimental observations can be summarized as follows:

1. Like in a permanent magnetic field, the field-induced phase separation in the rotating field is manifested through the appearance of needle-like dense particle aggregates whose size progressively increases with time via aggregate growth and coalescence mechanisms. Two fundamental differences are that the aggregate growth could be enhanced through convection of nanoparticles relatively to rotating aggregates, and the maximal aggregate size is limited by the fragmentation arising as a result of their hydrodynamic interactions/collisions.

2. The average aggregate length evolves between the lower limit probably dictated by the critical nuclei size and the upper limit dictated by the collision-induced fragmentation. The critical nuclei size and the aggregation threshold $H\left(\varphi^{\prime}\right)$ appear to be nearly independent of the rotational frequency of aggregates, $5 \leq v \leq 25 \mathrm{~Hz}$. On the contrary, the maximal aggregate size strongly decreases with the frequency approximately as $L_{\max } \propto v^{-2}$. Most of the 
aggregates perform a collective rotation in the same horizontal plane between the lower and the upper bounding walls.

3. The aggregate growth and the coalescence occur at similar timescales, $\tau_{d} \sim \tau_{c} \sim 1 \mathrm{~min}$, weakly dependent on the field frequency within the considered range $5 \leq v \leq 25 \mathrm{~Hz}$. This implies that the aggregate concentration $\Phi$ in the suspension continuously grows with time and the aggregate number density $n$ decreases with time, both showing a plateau at long times when the average size achieves its maximal value.

4. The experimental aggregate size distribution is found to significantly spread with time always keeping a non-negligible fraction of small aggregates, which is reminiscent for classical coagulation [Levich (1962); Aldous (1999)] or condensation-driven aggregation [Hassan and Hassan (2008)] processes. The fundamental difference is that in our case, we achieve a steadystate with finite size distribution, while classical coagulation leads to infinite aggregate growth.

The aforementioned experimental features are correctly reproduced by the developed theoretical models. In particular, the kinetics of aggregation beyond the nucleation stage is described by the population balance equation (2), simultaneously including the aggregate growth and coalescence mechanisms, while the critical nuclei size is used as the initial condition. The aggregate growth rate $\dot{V}$ is evaluated through the cavity model coupled with a diffusive boundary layer approach. The coalescence kernel is evaluated through the cell model, and the constant kernel approximation, $K\left(V, V^{\prime}\right) \approx$ const $=K_{0}$ is shown to approximately hold for our experimental conditions. The aggregate fragmentation is implicitly taken into account by allowing the aggregate growth rate and the coalescence constant decrease with increasing average volume as $\dot{V} \propto(1-\langle v\rangle)$ and $K=K_{0}(1-\langle v\rangle)$. All these approximations along with the linearization of the aggregate growth rate, $\dot{V} \propto V$, allow the analytical solution of the population balance equation, which gives satisfactory quantitative agreement with experiments on the average aggregate length $\langle L\rangle$ without any adjustable parameter and qualitatively reproduces the shape and the time behavior of the experimental length distribution function. Qualitatively, the frequency of the magnetic field affects the phase separation through (a) the maximal aggregate size $L_{\max } \propto v^{-2}$ (also confirmed by our theory) and (b) the convectiondiffusion timescale $t_{c o n v}=t_{d}\left(1+8\left(\ln \left(2 r_{\max }\right)-1 / 2\right)^{1 / 3} P e_{\max }^{-1 / 3} / k\right)$ depending on the Peclet number, $P e_{\max } \propto v$. The effect (b) appears to be small in the considered frequency range.

The current work can be useful for biomedical applications employing magnetic nanoparticles excited by rotating magnetic fields. One of the excellent examples is the noninvasive treatment of thromboses through the generation of blood recirculation near the blood clots by rotating nanoparticle aggregates [Creighton (2012)]. The current work allows evaluation of the aggregation timescale that must be fast enough with respect to the duration of the medical intervention.

\section{Supplementary Material}

Videos showing fast nanoparticle aggregation at first 5 seconds after application of the magnetic field (video-a.mp4) and the coalescence and sometimes fragmentation of aggregates at the timelapse $5-13 \mathrm{~s}$ after switching on the magnetic field (video-b.mp4). In both videos, the magnetic 
field amplitude and frequency are $9 \mathrm{kA} / \mathrm{m}$ and $5 \mathrm{~Hz}$, respectively and the nanoparticle volume fraction is $1.6 \times 10^{-3}$.

\section{Acknowledgements}

We are grateful to Dr. A. Bee and Dr. D. Talbot from PHENIX laboratory at Sorbonne University (Paris, France) for providing us with the parent ferrofluid. PK acknowledges the French 'Agence Nationale de la Recherche', Project Future Investments UCA JEDI, No. ANR15-IDEX-01 (projects ImmunoMag and MagFilter) and the private company Axlepios Biomedicals for financial support, JQC acknowledges the financial support of UCA JEDI and Axlepios Biomedicals through the PhD fellowship. AZ thanks the Russian Science Foundation, project 20-12-00031, for the financial support.

\section{AIP Publishing Data Sharing Policy}

The data that support the findings of this study are available within the article and its supplementary material.

\section{Appendix A. Aggregate magnetic susceptibility $\chi$ and internal volume fraction $\varphi^{\prime \prime}$}

Semi-empirical evaluation of the magnitudes $\chi$ and $\varphi^{\prime \prime}$ is performed by measuring the velocity of the aggregate migration under a magnetic field gradient and fitting it to a theoretical relationship developed below. These experiments are impossible in permanent magnetic fields because of gravitational settling of aggregates. However, in rotating fields, settling has never been observed (very likely due to hydrodynamic interactions with walls), and this allows evaluation of $\chi$ and $\varphi^{\prime \prime}$ in the conditions close to those realized in the phase separation experiments. To realize circularly polarized rotating magnetic field with a non-zero gradient, we have used the experimental setup shown on Fig. 3a, in which we have disconnected one of the internal coils. The pair of external coils and one connected internal coil were independently powered with AC voltage, using the procedure described in Sec. III-B. The amplitude and phase lag of two signals were adjusted in a way to ensure circularly polarized rotating magnetic field at a desired amplitude $\left(H_{0}=9 \mathrm{kA} / \mathrm{m}\right)$ and frequency $(v=5 \mathrm{~Hz})$ in the vicinity of the geometric center of the system. The three-coil configuration allowed obtaining the magnetic force on the superparamagnetic aggregates with the dominant component $F_{m, y}$ oriented along the $y$-axis of symmetry of the internal coils, as confirmed by analysis of Maxwell magnetostatic equations.

The microfluidic channel [Sec. III-B] was placed into the center of the system and oriented along the $y$-axis. The channel was filled with a dilute magnetic colloid subjected to salt/acid treatment (Sec. III-A) at a nanoparticle volume fraction $\varphi_{0}=1.6 \times 10^{-3}$. The applied nonuniform rotating field made the aggregates rotate synchronously with the field and migrate along the $y$-axis. We have not observed any substantial deviation of the trajectory of the aggregate centers from the $y$ direction. The aggregate motion was recorded by the CMOS camera and the linear velocity $v_{y}$ of their centers was measured using the Fiji image calculator. For data treatment, we have chosen a series of ten aggregates respecting the following criteria: (a) large enough to avoid visible aggregate growth for the time-lapse necessary for the velocity determination; (b) situated far enough from other aggregates and lateral walls to minimize 
possible hydrodynamic interactions. Let us now relate the measured aggregate velocity $v_{y}$ to its magnetic susceptibility $\chi$ under the linear magnetization assumption reasonable for relatively small applied magnetic field $H_{0}=9 \mathrm{kA} / \mathrm{m}$.

Since the aggregates are mostly symmetrical bodies (having a needle-like shape), their rotational and translational motion is uncoupled [Happel and Brenner (1983)]. As in the kinetic model [Sec. II], the aggregate shape is supposed to be a prolate ellipsoid with the major and minor semi-axes $a$ and $b$ and the aspect ratio $r_{a}=a / b$. It is also shown that in experimental conditions the phase lag between the orientations of the aggregate and the field is negligible [Sec. II-A], so the aggregates are supposed to be always aligned with the instantaneous direction of the field. In average (over the rotation period), the aggregates do not move across the channel, while the instantaneous velocity along the channel ( $y$ axis) is

$v_{y}(\theta)=\tilde{b}_{y y} F_{m, y}=\left(\tilde{b}_{\|} \cos \theta+\tilde{b}_{\perp} \sin \theta\right) \frac{1}{2} \mu_{0} \frac{\chi}{1+N \chi} \frac{\partial H_{0 y}^{2}}{\partial y} V \cos ^{2} \theta$,

$N=\frac{1}{2 r_{a}^{2}\left(1-r_{a}^{-2}\right)^{3 / 2}}\left[\ln \frac{1+\left(1-r_{a}^{-2}\right)^{1 / 2}}{1-\left(1-r_{a}^{-2}\right)^{1 / 2}}-2\left(1-r_{a}^{-2}\right)^{1 / 2}\right]$

$\tilde{b}_{\|}=\frac{1}{\eta_{0} f_{\|} a}, \tilde{b}_{\perp}=\frac{1}{\eta_{0} f_{\perp} a}, f_{\|}=\frac{16 \pi}{r_{a}^{2}\left(2 \beta \prime+\alpha_{\|}\right)}, f_{\perp}=\frac{16 \pi}{2 r_{a}^{2} \beta^{\prime}+\alpha_{\perp}}, \alpha_{\|}=\frac{2\left(\beta r_{a}^{2}-1\right)}{r_{a}^{2}-1}, \alpha_{\perp}=\frac{r_{a}^{2}(1-\beta \prime)}{r_{a}^{2}-1}, \beta^{\prime}=$ $\frac{\operatorname{acosh} r_{a}}{r_{a}\left(r_{a}^{2}-1\right)^{1 / 2}}$

where $H_{0 y}$ is the $y$-component of the amplitude of the oscillating magnetic field generated by the single powered internal coil; $N$ is the aggregate demagnetization factor [Landau and Lifshitz (1984)], $\theta=\omega t$ is the angle between the aggregate and the $y$ axis at a given moment of time $t$, $\tilde{b}_{y y}$ is the diagonal component of the aggregate mobility tensor along the $y$ axis of the channel, and $\tilde{b}_{\|}, \tilde{b}_{\perp}$ - the components of the mobility tensor parallel to the major and minor aggregate axes, respectively [Brenner (1974)]; other physical quantities are defined in the main text of the paper. Assuming homogeneous aggregate rotation, the $y$-component of the aggregate speed averaged over the rotation period (after some transformations) reads:

$$
\left\langle v_{y}\right\rangle=\frac{1}{2 \pi} \int_{0}^{2 \pi} v_{y}(\theta) d \theta=\frac{\pi}{48} \mu_{0} \frac{\chi}{1+N \chi} \frac{\partial H_{0 y}^{2}}{\partial y} \frac{(2 b)^{2}}{\eta_{0}}\left(\frac{3}{f_{\|}}+\frac{1}{f_{\perp}}\right),
$$

where the coefficients $N, f_{\|}$and $f_{\perp}$ are related to the aggregate aspect ratio $r_{a}$ by Eqs. (A1b), (A1c). The aggregate thickness $2 b$ and aspect ratio $r_{a}$ are determined by the direct size measurement on each aggregate, the value $\partial H_{0 y}^{2} / \partial y \approx 1.5 \times 10^{9} \mathrm{~A}^{2} / \mathrm{m}^{3}$ is found by magnetic field measurements coupled with the simulation of the field distribution, and $\eta_{0} \approx 10^{-3} \mathrm{~Pa} \times \mathrm{s}$ is the suspending liquid (water) viscosity.

The aggregate magnetic susceptibility $\chi$ is determined by fitting the experimental values of $\left\langle v_{y}\right\rangle$ to Eq. (A2). For the series of ten measurements, we get $\chi=22 \pm 5$. This value is consistent with the one, $\chi \approx 24$, evaluated experimentally by Bacri et al. (1994) in experiments with elongation of the magnetic droplet. 
The nanoparticle volume fraction $\varphi^{\prime \prime}$ inside the aggregates can be retrieved from the $\chi$ value using some statistical mechanics model of the ferrofluid microstructure. First, we presume that when the primary agglomerates ( 20-100 nm, cf. Fig. 2) form bulk needle-like aggregates under an applied magnetic field, the individual nanoparticles $(\sim 8 \mathrm{~nm})$ reorganize inside these bulk aggregates and form a dense phase but not compulsorily a close packing. Secondly, nanoparticles have a relatively narrow size distribution, as revealed by magnetization measurements [Sec. III-A]. At these two conditions, a modified MMF2 model of Szalai et al. (2015), developed for concentrated (up to $40 \% \mathrm{vol}$ ) monodisperse colloid seems to be an adequate candidature for our case. According to this model, the aggregate magnetic susceptibility is

$\chi=\chi_{L}\left[1+\frac{1}{3} \chi_{L}+\frac{1}{144} \chi_{L}^{2} f_{L}\left(\varphi^{\prime \prime}\right)\right], \chi_{L}=8 \varphi^{\prime \prime} \lambda_{d}, \quad \lambda_{d}=\frac{\pi \mu_{0} M_{S}^{2}}{144 k_{B} T} \frac{\left\langle d_{m}^{6}\right\rangle}{\left\langle d_{m}^{3}\right\rangle}$,

$f_{L}\left(\varphi^{\prime \prime}\right)=17 \frac{1-0.93952 \rho+0.36714 \rho^{2}}{1-0.92398 \rho+0.23323 \rho^{2}}-16, \rho=\frac{6 \varphi \prime \prime}{\pi}$.

Here, $\chi_{L}$ is the Langevin magnetic susceptibility (in the absence of interactions between particles), $\lambda_{d}$ is the dipolar coupling parameter, $M_{S} \approx 3.1 \times 10^{5} \mathrm{~A} / \mathrm{m}-$ saturation magnetization of nanoparticles, retrieved from magnetization measurements [Sec. III-A], $\left\langle d_{m}^{3}\right\rangle$ and $\left\langle d_{m}^{6}\right\rangle$ are the moments of the log-normal size distribution function [Eq. (38)], whose values are given below Eq. (39).

Solving numerically the transcendental equation (A3) with respect to $\varphi^{\prime \prime}$, we get the following value of the nanoparticle volume fraction inside the aggregates: $\varphi^{\prime \prime}=0.32 \pm 0.03$. This value is consistent with the one $\left(\varphi^{\prime \prime} \approx 0.3\right)$ evaluated experimentally by Sandre et al. (1999). Notice that for most precise evaluations of $\chi$ and $\varphi^{\prime \prime}$ we used exact expressions for the demagnetization factor [Eq. (A1b)] and hydrodynamic mobility [Eq. (A1c)] instead of approximate expressions at high aspect ratio limit adopted in the kinetic model [Sec. II].

\section{Appendix B. Aggregate shape and surface tension}

Our kinetic model [Sec. II] requires explicit relationships between the aggregate aspect ratio, thickness, length and volume. It is commonly admitted that these relationships come from the condition of the free energy minimum [Blums et al. (1997); Zubarev and Ivanov (1997)]; which applies even in rotating fields at relatively low frequency when the hydrodynamics modifies only slightly the local thermodynamic equilibrium of aggregates, as shown in Sec. IV-B. As in the kinetic model [Sec. II], the aggregate shape is supposed to be a prolate ellipsoid with the major and minor semi-axes $a$ and $b$ and the aspect ratio $r_{a}=a / b$. Under considered linear magnetization approximation, the free energy for such aggregate reads:

$$
\begin{aligned}
& \mathcal{F}=-\frac{1}{2} \mu_{0} M_{a} H_{0} V+\sigma S, \\
& M_{a}=\frac{\chi H_{0}}{1+N \chi}, \\
& S=V^{2 / 3} s, \quad s=2 \pi\left(\frac{3}{4 \pi}\right)^{2 / 3} r_{a}^{-2 / 3}\left(1+\frac{r_{a} a \sin \left(1-r_{a}^{-2}\right)^{1 / 2}}{\left(1-r_{a}^{-2}\right)^{1 / 2}}\right),
\end{aligned}
$$


where $\sigma$ and $S$ are the aggregate surface tension and surface area, respectively, while $M_{a}$ and $\chi$ are the aggregate magnetization and magnetic susceptibility, respectively $(\chi=22 \pm 5$, as determined in Appendix A); the aggregate demagnetization factor $N$ is given by Eq. (A1b) as a function of the aggregate aspect ratio $r_{a}$.

The surface tension $\sigma$ is usually an unknown parameter, but it can be obtained by fitting the experimental aggregate shape to the modelled one. In our case where attractive van der Waals interaction between nanoparticles could play an important role, it is reasonable to suppose that the surface tension has one term depending on magnetization and the second constant term appearing due to non-magnetic colloidal interactions between nanoparticles:

$$
\sigma=\sigma_{0}+\mu_{0} M_{a}^{2} \delta
$$

where $\delta$ is the characteristic thickness of the transition surface layer, which is typically on the order of the average size $\left\langle d_{m}\right\rangle$ of individual magnetic nanoparticles. The energy minimization with respect to the aggregate aspect ratio $r_{a}$ at a fixed aggregate volume $V$ and external magnetic field amplitude $H_{0}$ gives the following expression:

$$
\left(\frac{\partial \mathcal{F}}{\partial r_{a}}\right)_{V, H_{0}=\text { const }}=\frac{1}{2} \mu_{0} M_{a}^{2} V \frac{d N}{d r_{a}}+\sigma V^{2 / 3} \frac{d s}{d r_{a}}-2 \mu_{0} M_{a}^{2} V^{2 / 3} \delta \frac{\chi}{1+N \chi} \frac{d N}{d r_{a}} s=0,
$$

where the last term comes from the derivative of the surface tension [Eq. (B2)] (or rather its term depending on the magnetization) with respect to the aspect ratio; this term is usually not taken into account in the classical models [Blums et al. (1997); Zubarev and Ivanov (1997)]. Solving Eq. (B3) with respect to $V$, using Eqs. (B1b) and (B2), one obtains the following relationship between the aggregate volume and aspect ratio:

$V=(2 \delta)^{3}\left[-\frac{d N / d r_{a}}{d s / d r_{a}}\left(1+\frac{\sigma_{0}}{\mu_{0} H_{0}^{2} \delta}\left(\frac{1+N \chi}{\chi}\right)^{2}\right)+2 s \frac{\chi}{1+N \chi}\right]^{3}$,

with $N$ and $s$ given by Eqs. (A1b) and (B1c) as functions of $r_{a}$. In high aspect ratio limit, relevant for the present study, the last equation reduces to

$V=\left\{\begin{array}{c}\frac{\pi^{4} \delta^{3}}{48}\left[\frac{r_{a}^{7 / 3}}{\ln \left(2 r_{a}\right)-3 / 2}\left(1+\frac{\sigma_{0}}{\mu_{0} \chi^{2} H_{0}^{2} \delta}\right)+12 \chi r_{a}^{1 / 3}\right]^{3}, \text { at } r_{a}^{2} \sim 12 \chi \gg 1, \\ \frac{\pi^{4}}{48}\left(1+\frac{\sigma_{0}}{\mu_{0} \chi^{2} H_{0}^{2} \delta}\right)^{3} \frac{r_{a}^{7}}{\left(\ln \left(2 r_{a}\right)-3 / 2\right)^{3}}, \text { at } r_{a}^{2} \gg 12 \chi \text { and } r_{a}^{2} \gg 1, \\ 36 \pi^{4} \chi^{3} \delta^{3} r_{a}, \text { at } 12 \chi \gg r_{a}^{2} \gg 1\end{array}\right.$

The two unknown parameters, the first term $\sigma_{0}$ of the aggregate surface tension and the surface layer thickness $\delta$ are determined by fitting the experimental $V\left(r_{a}\right)$ dependencies (obtained by image processing of our kinetic experiments - see for instance Fig. 4) to the theoretical dependencies [Eqs. (B4) or (B5)]. This fit is shown in Fig. 10 for several series of experimental data. The figure shows that, because of some dispersion of data, it is quite difficult to clearly see the effect of the magnetic field amplitude $H_{0}$ and frequency $v$ on experimental $V\left(r_{a}\right)$ dependencies. The theory [Eq. (B4)] also predicts only a slight shift of the $V\left(r_{a}\right)$-dependency with the change in magnetic field amplitude $H_{0}$ - see the black solid and blue dashed curves 
for $H_{0}=9$ and $6 \mathrm{kA} / \mathrm{m}$, respectively. The best fit by Eq. (B4) is obtained for the following values of the adjustable parameters: $\delta=12 \mathrm{~nm}$ and $\sigma_{0}=1.5 \times 10^{-4} \mathrm{~N} / \mathrm{m}$. As expected, the first value is close to the average nanoparticle diameter $\left\langle d_{m}\right\rangle=8.1 \mathrm{~nm}$ [Sec. III-A], while the second one is by two orders of magnitude larger than the one $\left(\sigma_{0}=2.8 \times 10^{-6} \mathrm{~N} / \mathrm{m}\right)$ found by Bacri et al. (1994).

Notice that the classical model [Zubarev and Ivanov (1997)], neglecting the variation of the surface tension with $r_{a}$ (last term in brackets of Eq. (B4)), corresponds to the limit $r_{a}^{2} \gg$ $12 \chi$ of our model. This limit provides the behavior $V \propto r_{a}^{7}$, that is reproduced by the second expression in Eq. (B5) of the high aspect ratio limit of our model. It is found that the classical model is not able to fit even approximately the experimental data on Fig. 10. On the other hand, at not too high aspect ratios $r_{a} \lesssim 10$, relevant for our experiments, we are in the opposite limit $12 \chi \gg r_{a}^{2} \gg 1$, for which the aggregate volume is predicted to be roughly linear with aspect ratio, $V \propto r_{a}$, as inferred from the third expression of Eq. (B5). The theoretical $V\left(r_{a}\right)$ dependency corresponding to this limit is shown by a dotted red line in Fig. 10. For the sake of comparison, we have used the same value $\delta=12 \mathrm{~nm}$ of the transition layer thickness for this dependency. This simple linear law seems to agree with experimental data only at aspect ratios $r_{a} \lesssim 8$. However, this approximation gives a good agreement on the aggregate half-thickness $b$ that does not change with the magnetic field amplitude and frequency both in experiments and in theory. In the considered limit $12 \chi \gg r_{a}^{2} \gg 1$, the $b$ value is easily obtained from the third expression of Eq. (B5) using the obvious geometrical relationship $V=4 \pi b^{3} r_{a} / 3$ :

$$
b \approx 3 \pi \chi \delta \approx \text { const }
$$

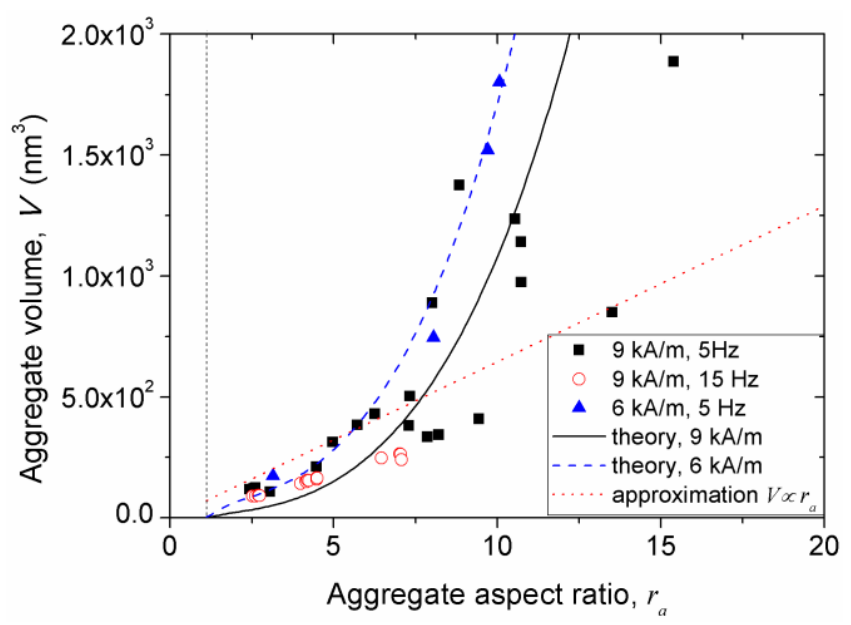

Fig. 10. Experimental dependencies (symbols) of the aggregate volume on its aspect ratio. The solid black and the dashed blue curves correspond to the fit of experimental data by Eq. (B4) with the adjustable parameters $\delta=$ $12 \mathrm{~nm}$ and $\sigma_{0}=1.5 \times 10^{-4} \mathrm{~N} / \mathrm{m}$. The dotted red line corresponds to the prediction of the limit $12 \chi \gg r_{a}^{2} \gg 1$ [third expression in Eq. (B5)] at $\delta=12 \mathrm{~nm}$. The vertical dashed line corresponds to the aspect ratio $r_{a}=1$.

The evaluation gives $b \approx 2.5 \mu \mathrm{m}$ at $\delta=12 \mathrm{~nm}$ and $\chi \approx 22$ [Appendix A] in a perfect agreement with experiments. In summary, the present model of the aggregate shape/surface tension allows a correct agreement with experiments and covers the two limiting behaviors: $V \propto r_{a}, \quad b \approx$ const,$a \propto r_{a} \propto V$ at $r_{a}^{2} \ll 12 \chi$ and $V \propto r_{a}^{7}, b \propto r_{a}^{2} \propto V^{2 / 7}, a \propto r_{a}^{3} \propto V^{3 / 7}$ at 
$r_{a}^{2} \gg 12 \chi$, with the second limit being commonly admitted in literature [Zubarev and Ivanov (1997); Ezzaier et al. (2017)].

Appendix C. Hydrodynamic coefficient $A_{f}[\mathrm{Eq} \cdot(12 \mathrm{~b})]$ and hydrodynamic pressure.

Within the thin diffusive boundary layer around the aggregate, the velocity field in the reference frame of the aggregate [see the magnified view of a single aggregate on the right of Fig. 1a] is supposed to take a functional form as in the case of the creeping flow past a cylinder. In polar coordinates $(\rho, \theta)$ introduced in Fig. 1a, it reads [Adamczyk and Van de Ven (1981)]:

$$
v_{\theta}=4 A_{f} u_{\infty} \frac{\rho-r}{r} \sin \theta, \quad v_{\rho}=-2 A_{f} u_{\infty}\left(\frac{\rho-r}{r}\right)^{2} \cos \theta,
$$

where $r$ is the radial coordinate of the aggregate surface at an axial position $z$, and the velocity at the infinity is set to $u_{\infty}=\omega z$. The hydrodynamic coefficient $A_{f}$ in the present case of a rotating ellipsoidal aggregate is expected to be different from that for the flow past un infinite cylinder. The value of $A_{f}$ for a given axial position $z$ can be easily obtained by comparing the shear rate near the aggregate surface

$$
\frac{\partial v_{\theta}}{\partial \rho}=4 A_{f} \frac{\omega z}{r} \sin \theta
$$

given by the velocity field (C1) with the shear rate occurring on the surface of rotating ellipsoidal aggregate, for which the velocity field is given by the Jeffery solution [Jeffery (1922)]. After some transformations, the expressions for the transverse velocity components in the cartesian reference frame of the aggregate read:

$$
\begin{aligned}
& v_{x}=-\frac{4 x y z \Pi \varepsilon}{\left(b^{2}+\lambda\right)^{3}\left(a^{2}+\lambda\right)^{3 / 2}}\left[\left(b^{2}+\lambda\right) a^{2}-\left(a^{2}+\lambda\right) b^{2}\right], \\
& v_{y}=\omega z-2 z \varepsilon\left(\gamma a^{2}+\beta b^{2}\right)-\frac{4 y^{2} z \Pi \varepsilon}{\left(b^{2}+\lambda\right)^{3}\left(a^{2}+\lambda\right)^{3 / 2}}\left[\left(b^{2}+\lambda\right) a^{2}-\left(a^{2}+\lambda\right) b^{2}\right], \\
& \Pi(\lambda)=\left[\frac{x^{2}+y^{2}}{\left(b^{2}+\lambda\right)^{2}}+\frac{z^{2}}{\left(a^{2}+\lambda\right)^{2}}\right]^{-1}, \quad \varepsilon=\frac{\omega}{2\left(b^{2} \beta_{0}+a^{2} \gamma_{0}\right)}, \\
& \beta(\lambda)=\int_{\lambda}^{\infty} \frac{d \lambda}{\left(b^{2}+\lambda\right)^{2}\left(a^{2}+\lambda\right)^{1 / 2}}, \gamma(\lambda)=\int_{\lambda}^{\infty} \frac{d \lambda}{\left(b^{2}+\lambda\right)\left(a^{2}+\lambda\right)^{3 / 2}}, \\
& \beta_{0}=\beta(0)=b^{-3}\left[\frac{r_{a}}{r_{a}^{2}-1}-\frac{\operatorname{acosh} r_{a}}{\left(r_{a}^{2}-1\right)^{3 / 2}}\right], \quad \gamma_{0}=\gamma(0)=-2 b^{-3}\left[\frac{1}{r_{a}\left(r_{a}^{2}-1\right)}-\frac{\operatorname{acosh} r_{a}}{\left(r_{a}^{2}-1\right)^{3 / 2}}\right],
\end{aligned}
$$

with the ellipsoidal coordinate $\lambda$ being related to the cartesian coordinates by

$$
\frac{x^{2}+y^{2}}{b^{2}+\lambda}+\frac{z^{2}}{a^{2}+\lambda}=1
$$

Notice that $\beta$ in Eq. (C3) should not be confounded with the constant $\beta$ used in the population balance equation [Eq. (25)]. Expanding both velocity components into series around $\lambda=0$ and keeping only the linear term on $\lambda$, we get the following expressions for the fluid velocity in the vicinity of the aggregate surface: 
$v_{x}=\frac{4\left(b^{2}-a^{2}\right) x y z \Pi_{0} \varepsilon}{b^{6} a^{3}} \lambda, \quad v_{y}=\frac{4 z \varepsilon}{b^{2} a} \lambda+\frac{4\left(b^{2}-a^{2}\right) y^{2} z \Pi_{0} \varepsilon}{b^{6} a^{3}} \lambda, \quad \Pi_{0}=\Pi(0)=\left(\frac{x^{2}+y^{2}}{b^{4}}+\frac{z^{2}}{a^{4}}\right)^{-1}$.

The shear rate on the ellipsoid surface $(\lambda=0)$ is evaluated as follows:

$\left(\frac{\partial v_{\theta}}{\partial \rho}\right)_{\lambda=0}=-\left(\frac{\partial v_{x}}{\partial \rho}\right)_{\lambda=0} \cos \theta+\left(\frac{\partial v_{y}}{\partial \rho}\right)_{\lambda=0} \sin \theta=\frac{8 \Pi_{0} \varepsilon}{b^{4} a} r z \sin \theta$,

where we have used the following relationships:

$x=-\rho \sin \theta, \quad y=-\rho \cos \theta, \quad x^{2}+y^{2}=\rho^{2}, \quad\left(\frac{\partial \lambda}{\partial \rho}\right)_{\lambda=0}=\frac{2 \Pi_{0} \rho}{b^{2}}$.

In high aspect ratio limit, $r_{a} \gg 1$, the equation (C5) reduces to

$$
\left(\frac{\partial v_{\theta}}{\partial \rho}\right)_{\lambda=0} \approx \frac{2}{\ln \left(2 r_{a}\right)-1 / 2} \frac{\omega z}{r} \frac{\hat{r}^{2}}{\hat{r}^{2}+\hat{z}^{2} / r_{a}^{2}} \sin \theta,
$$

with $\hat{r}=r / b$ and $\hat{z}=z / a$ being the dimensionless coordinates of the aggregate surface. Equalizing the right-hand parts of Eqs. (C2) and (C6), we arrive at Eq. (12b) for the hydrodynamic coefficient $A_{f}$.

Let us now evaluate the hydrodynamic pressure on the surface of an ellipsoidal aggregate spinning in a quiescent suspending liquid at angular frequency $\omega$ [Fig. 1a]. According to Jeffery (1922), at any point of the suspending fluid, the hydrodynamic pressure reads:

$$
p_{H}=-\frac{8 \eta_{0} y z \Pi \varepsilon\left(a^{2}-b^{2}\right)}{\left(b^{2}+\lambda\right)^{2}\left(a^{2}+\lambda\right)^{3 / 2}} .
$$

At the aggregate surface, $\lambda=0$, under high aspect ratio limit, $r_{a} \gg 1$, the last equation reduces to:

$$
p_{H} \approx-\frac{2 \eta_{0} \omega}{\ln \left(2 r_{a}\right)-1 / 2} \frac{y z}{b^{2}}
$$

The pressure takes its maximal absolute value at the points on the aggregate surface situated at $x=0, y= \pm b / \sqrt{2}, z=a / \sqrt{2}$ :

$$
\left|p_{H, \max }\right|=\frac{\eta \omega r_{a}}{\ln \left(2 r_{a}\right)-1 / 2} .
$$

This value is used as a characteristic hydrodynamic pressure on the aggregate surface.

\section{Appendix D. Diffusive flux towards the cavity [Eq. (14)].}

We consider the nanoparticle diffusion from infinity where the nanoparticle volume fraction is $\varphi$ towards the cavity, representing an oblate ellipsoid of the semi-axes $b$ and $a$ [Fig. 1c]; the nanoparticle volume fraction on the cavity surface is $\varphi_{c}<\varphi$. The diffusion problem is considered in the ellipsoidal reference frame $(\xi, \eta, \phi)$ [Fig. 1c] related to the cylindrical reference frame $(\rho, z, \phi)$ as follows [Happel and Brenner (1983)]:

$$
z=c \sinh \xi \cos \eta, \quad \rho=c \cosh \xi \sin \eta, \quad \phi=\phi,
$$


where the length scale $c=\left(a^{2}-b^{2}\right)^{1 / 2}$ should not be confounded with the numerical constant $c \approx 3.644$ in Eq. (12a).

In the considered boundary conditions, the particle volume fraction is constant on the surfaces of confocal oblate ellipsoids, thus, only depends on the coordinate $\xi$ describing the position of these ellipsoids [Fig. 1c]. The surface flux density vector is everywhere perpendicular to these surfaces and its single non-zero $\xi$-component reads:

$$
j_{\xi}=-D_{p} \nabla_{\xi} \varphi=-\frac{D_{p}}{c\left(\cosh ^{2} \xi-\sin ^{2} \eta\right)^{1 / 2}} \frac{d \varphi}{d \xi}
$$

The volume nanoparticle flux across any coordinate surface $\xi=$ const is obtained by integration of the flux density:

$J_{V 1}=-\oint j_{\xi} d S=-2 \pi \int_{0}^{\pi} j_{\xi} c^{2}\left(\cosh ^{2} \xi-\sin ^{2} \eta\right)^{1 / 2} \cosh \xi \sin \eta d \eta=4 \pi D_{p} c \cosh \xi \frac{d \varphi}{d \xi}$,

where the minus sign at the integral indicates that the nanoparticle flux towards the cavity is opposed to the outward direction of the $\xi$ axis. Isolating $d \varphi$ and integrating over $\xi$ from the cavity surface to the infinity, we get:

$$
\varphi-\varphi_{c}=\int_{\xi_{0}}^{\infty} \frac{J_{V 1}}{4 \pi D_{p} c} \frac{d \xi}{\cosh \xi}=\frac{J_{V 1}}{4 \pi D_{p} c}\left(\pi-2 \operatorname{atan}\left(e^{\xi_{0}}\right)\right)
$$

where the volume flux $J_{V 1}$ is considered to be constant in the conventional approximation of quasi-stationary diffusion and $\xi_{0}=\operatorname{atanh}(b / a)=\operatorname{atanh}\left(r_{a}{ }^{-1}\right)$ is the value of $\xi$ at the cavity surface. The expression for $J_{V 1}$ as a function of the volume fraction difference $\varphi-\varphi_{c}$ directly follows form Eq. (D3):

$$
J_{V 1}=\frac{4 \pi D_{p}\left(a^{2}-b^{2}\right)^{1 / 2}}{\pi-2 \operatorname{atan}\left(e^{\operatorname{atanh}\left(r_{a}^{-1}\right)}\right)}\left(\varphi-\varphi_{c}\right)
$$

For high aspect ratio aggregates $r_{a} \gg 1$, the cavity is a very oblate ellipsoid and the equation (D4) reduces to Eq. (14).

\section{Appendix E. Coagulation kernel.}

To evaluate the flux of the neighboring aggregates towards the target aggregate, let us consider two slender aggregates of the volumes $V$ and $V^{\prime}$, semi-lengths $a$ and $a^{\prime}$ and aspect ratios $r_{a}$ and $r_{a}{ }^{\prime}$ rotating in the same horizontal plane, as observed in experiments and shown in Fig. 1d. Recall that the aggregate half-thickness is nearly independent of the volume: $b \approx b^{\prime} \approx$ const [Eq. (B6)]. The origin of the Cartesian $(\rho, z)$ and polar $(r, \theta)$ reference frames is placed into the center of the target aggregate $\left(V, a, r_{a}\right)$, and the $z$ axis with $\theta=0$ is aligned with the major axis of the target aggregate. The flux $J_{V \rightarrow V}$ of the neighboring aggregates towards the target aggregate could be evaluated with the help of the cell model widely used for calculation of mechanical, thermal or optical properties of composite materials [Christensen (1979); Berthier (1993)]. According to this model, individual structural units (aggregates in our case) are surrounded by representative cells with appropriately chosen boundary conditions on the 
cell surface. Since all the aggregates perform a homogeneous rotation in the same horizontal plane, it is reasonable to choose a disk-like shape for the representative cells with the axis of symmetry perpendicular to the rotation plane and passing through the aggregate centers [Fig. $1 \mathrm{~d}]$. The cell thickness is equal to the vertical gap $h$ of the sample and its volume, $V_{\text {cell }}$ or $V^{\prime}$ cell is chosen to respect the volume fraction $\Phi=V / V_{\text {cell }}=V^{\prime} / V^{\prime}$ cell of the aggregates in the colloid. The cell radii can be easily evaluated from geometrical considerations:

$$
R=\left(\frac{V}{\pi h \Phi}\right)^{1 / 2}=2 b\left(\frac{a}{3 h \Phi}\right)^{1 / 2}, R^{\prime}=\left(\frac{V^{\prime}}{\pi h \Phi}\right)^{1 / 2}=2 b\left(\frac{a^{\prime}}{3 h \Phi}\right)^{1 / 2} .
$$

The cells of neighboring aggregates touch the cell of the target aggregate, and the distance $r$ between the centers of two aggregates is equal to $r=R+R^{\prime}$. The small phase lag $\phi$ between the aggregates and the field orientation is neglected along with possible misalignment of two neighboring aggregates because of magnetic and hydrodynamic interactions between them. Thus, all the aggregates are supposed to be parallel to the instantaneous direction of the rotating field.

The flux $J_{V \rightarrow V}$, can be related to the components $F_{\rho}$ and $F_{Z}$ of the magnetic force acting between the target and the neighboring aggregate, as follows [Ezzaier et al. (2017)]:

$$
\begin{gathered}
J_{V \rightarrow V}=-n_{V}\left[\tilde{b}_{\|} \int F_{z} n_{z} d S+\tilde{b}_{\perp} \int F_{\rho} n_{\rho} d S\right], \\
\tilde{b}_{\|} \approx \frac{\ln \left(2 r_{a}\right)-\frac{1}{2}}{4 \pi \eta_{0} a}+\frac{\ln \left(2 r_{a^{\prime}}\right)-\frac{1}{2}}{4 \pi \eta_{0} a^{\prime}}, \quad \tilde{b}_{\perp} \approx \frac{\ln \left(2 r_{a}\right)+\frac{1}{2}}{8 \pi \eta_{0} a}+\frac{\ln \left(2 r_{a^{\prime}}\right)+\frac{1}{2}}{8 \pi \eta_{0} a^{\prime}}, n_{z}=\cos \theta, \quad n_{\rho}=\sin \theta, d S=h r d \theta
\end{gathered}
$$

where $\tilde{b}_{\|}$and $\tilde{b}_{\perp}$ are longitudinal and transverse components of the tensor of mutual hydrodynamic mobility of both aggregates, evaluated at high aspect ratio limit, $r_{a}, r_{a}{ }^{\prime} \gg 1$ [Brenner (1974)]; the integrals are taken over the lateral cylindrical surface $S$ of the excluded volume shell surrounding the target aggregate and having a radius equal to the distance $r$ between aggregates' centers; $n_{z}$ and $n_{\rho}$ are components of the unit outward vector normal to the surface $S$; the minus sign corresponds to the positive inward flux. Notice that we do not need to average the flux $J_{V \rightarrow V}$, over the all possible aggregate orientations because it is implicitly done by integration of the flux density over the surface $S$ of the excluded volume shell.

The magnetic force between aggregates can be evaluated in a non-point dipole limit, i.e. representing the aggregate magnetic moments $m_{a}=2 a q$ and $m_{a}{ }^{\prime}=2 a^{\prime} q^{\prime}$ as the dipole moment of two equivalent charges $+q$ and $-q$ (or $+q^{\prime}$ and $-q^{\prime}$ ) placed onto aggregate tips [Fig. 1d] and calculating the force between four pairs of charges belonging to two aggregates. A similar approach has been used in our previous work on the permanent magnetic field and equal-sized aggregates [Ezzaier et al. (2017)]. In the present case of the rotating field and unequal aggregates, we get:

$$
F_{\rho}=\frac{q q^{\prime}}{4 \pi \mu_{0} r^{2}}\left[\frac{\sin \theta}{\hat{r}_{N N}^{3}}+\frac{\sin \theta}{\hat{r}_{S S}^{3}}-\frac{\sin \theta}{\hat{r}_{N S}^{3}}-\frac{\sin \theta}{\hat{r}_{S N}^{3}}\right]
$$




$$
\begin{gathered}
F_{z}=\frac{q q^{\prime}}{4 \pi \mu_{0} r^{2}}\left[\frac{\cos \theta-\left(\hat{a}-\hat{a}^{\prime}\right)}{\hat{r}_{N N}^{3}}+\frac{\cos \theta+\left(\hat{a}-\hat{a}^{\prime}\right)}{\hat{r}_{S S}^{3}}-\frac{\cos \theta-(\hat{a}+\hat{a} \prime)}{\hat{r}_{N S}^{3}}-\frac{\cos \theta+\left(\hat{a}+\hat{a}^{\prime}\right)}{\hat{r}_{S N}^{3}}\right] \\
q=\frac{m_{a}}{2 a}=\frac{\mu_{0} M_{a} V}{2 a} \approx \frac{2 \pi}{3} \mu_{0} \chi H_{0} b^{2}=q^{\prime},
\end{gathered}
$$

$\hat{r}_{N N}^{2}=1+\left(\hat{a}-\hat{a}^{\prime}\right)^{2}-2\left(\hat{a}-\hat{a}^{\prime}\right) \cos \theta, \quad \hat{r}_{S S}^{2}=1+\left(\hat{a}-\hat{a}^{\prime}\right)^{2}+2\left(\hat{a}-\hat{a}^{\prime}\right) \cos \theta$,

$\hat{r}_{N S}^{2}=1+\left(\hat{a}+\hat{a}^{\prime}\right)^{2}-2\left(\hat{a}+\hat{a}^{\prime}\right) \cos \theta, \quad \hat{r}_{S N}^{2}=1+\left(\hat{a}+\hat{a}^{\prime}\right)^{2}+2\left(\hat{a}+\hat{a}^{\prime}\right) \cos \theta$,

where $\hat{r}_{i k}(i, k=N, S)$ are the distances between the $i^{\text {th }}$ pole of the target aggregate and the $k^{\text {th }}$ pole of the neighboring aggregate, normalized by the distance $r$ between the centers of both aggregates; the subscripts " $N$ " and " $S$ " stand for the north $(+q$ or $+q$ ') and south $(-q$ or $-q$ ') poles, respectively, $\hat{a}=a / r$ and $\hat{a}^{\prime}=a^{\prime} / r$ are the aggregates' half-lengths normalized by $r$; $M_{a} \approx M_{a}{ }^{\prime} \approx \chi H_{0}$ is the aggregate magnetization evaluated in high aspect ratio limit, $r_{a}, r_{a}{ }^{\prime} \gg$ 1 neglecting demagnetizing effects. Combining Eqs. (20), (E1) - (E3), after some transformations, we arrive at the following expression for the coagulation kernel:

$$
\begin{gathered}
K\left(V, V^{\prime}\right)=-\frac{\mu_{0} \chi^{2} H_{0}^{2} b^{2} h}{72 \eta_{0}} \frac{\hat{a}+\hat{a}^{\prime}}{r_{a}+r_{a^{\prime}}}\left[2 B_{-}\left(r_{a}, r_{a}{ }^{\prime}\right) X\left(\hat{a}, \hat{a}^{\prime}\right)+B_{+}\left(r_{a}, r_{a}{ }^{\prime}\right) Y\left(\hat{a}, \hat{a}^{\prime}\right)\right] \geq 0, \\
B_{ \pm}\left(r_{a}, r_{a}^{\prime}\right)=\frac{\ln \left(2 r_{a}\right) \pm 1 / 2}{r_{a}}+\frac{\ln \left(2 r_{a^{\prime}}\right) \pm 1 / 2}{r_{a^{\prime}}}, \\
X\left(\hat{a}, \hat{a}^{\prime}\right)=4 \int_{0}^{\pi / 2}\left(\frac{\cos \theta-\left(\hat{a}-\hat{a}^{\prime}\right)}{\hat{r}_{N N}^{3}}+\frac{\cos \theta+\left(\hat{a}-\hat{a}^{\prime}\right)}{\hat{r}_{S S}^{3}}-\frac{\cos \theta-\left(\hat{a}+\hat{a}^{\prime}\right)}{\hat{r}_{N S}^{3}}-\frac{\cos \theta+\left(\hat{a}+\hat{a}^{\prime}\right)}{\hat{r}_{S N}^{3}}\right) \cos \theta d \theta, \\
Y\left(\hat{a}, \hat{a}^{\prime}\right)=4 \int_{0}^{\pi / 2}\left(\frac{1}{\hat{r}_{N N}^{3}}+\frac{1}{\hat{r}_{S S}^{3}}-\frac{1}{\hat{r}_{N S}^{3}}-\frac{1}{\hat{r}_{S N}^{3}}\right) \sin ^{2} \theta d \theta, \\
\hat{a}=\frac{1}{2}\left(\frac{3 h \Phi}{b}\right)^{1 / 2} \frac{r_{a}}{r_{a}^{1 / 2}+\left(r_{a^{\prime}}\right)^{1 / 2}}, \hat{a}^{\prime}=\frac{1}{2}\left(\frac{3 h \Phi}{b}\right)^{1 / 2} \frac{r_{a^{\prime}}}{r_{a}^{1 / 2}+\left(r_{a^{\prime}}\right)^{1 / 2}},
\end{gathered}
$$

where the integrals in Eqs. (E4c), (E4d) are evaluated numerically. One can explicitly express the coagulation kernel through the aggregate volumes $V$ and $V^{\prime}$ by replacing $r_{a}$ and $r_{a}{ }^{\prime}$ in Eqs. (E4) by the second expression of Eq. (16). As expected, the coagulation kernel in Eq. (E4a) is symmetric with respect to volume permutation: $K\left(V, V^{\prime}\right)=K\left(V^{\prime}, V\right)$.

\section{References}

Adamczyk, Z., \& Van de Ven, T. G. M. (1981). Deposition of Brownian particles onto cylindrical collectors. Journal of Colloid and Interface Science, 84(2), 497-518.]

Aldous, D. J. (1999). Deterministic and stochastic models for coalescence (aggregation and coagulation): a review of the mean-field theory for probabilists. Bernoulli, 5(1), 3-48.

Bacri, J. C., Cebers, A. O., \& Perzynski, R. (1994). Behavior of a magnetic fluid microdrop in a rotating magnetic field. Physical review letters, 72(17), 2705.

Berret, J. F. (2016). Local viscoelasticity of living cells measured by rotational magnetic spectroscopy. Nature communications, 7(1), 1-9

Berthier S., Optics of Composite Media (in French: Optique des Milieux Composites) (1993) Polytechnica, Paris. Blums E., A. Cebers, and M. M. Mayorov (1977). Magnetic Fluids. Walter de Gruyters, Berlin.

Brenner, H. (1974). Rheology of a dilute suspension of axisymmetric Brownian particles. International journal of multiphase flow, 1(2), 195-341. 
Buyevich, Y. A., \& Ivanov, A. O. (1992). Equilibrium properties of ferrocolloids. Physica A, 190(3-4), $276-294$. Cébers, A. (2002). Dynamics of an elongated magnetic droplet in a rotating field. Physical Review E, 66(6), 061402.

Chiriac, H.; Radu, E.; Țibu, M.; Stoian, G.; Ababei, G.; Lăbuşcă, L.; Herea, D.-D.; Lupu, N. Fe-Cr-Nb-B Ferromagnetic Particles with Shape Anisotropy for Cancer Cell Destruction by Magnetomechanical Actuation. Sci. Rep. 2018, 8, 11538.

Christensen R. M., Mechanics of Composite Materials (1979) Krieger Publishing Company, Malabar.

Christensen, R. M. (1993). Effective viscous flow properties for fiber suspensions under concentrated conditions. Journal of Rheology, 37(1), 103-121.

Creighton, Francis M. 2012."Magnetic-based systems for treating occluded vessels." U.S. Patent No. 8,308,628. 13 Nov.

Cueille, S., \& Sire, C. (1997). Smoluchowski's equation for cluster exogenous growth. EPL (Europhysics Letters), 40(3), 239.

Dubois, E., Cabuil, V., Boué, F., \& Perzynski, R. (1999). Structural analogy between aqueous and oily magnetic fluids. The Journal of chemical physics, 111(15), 7147-7160.

Ezzaier, H., Alves Marins, J., Razvin, I., Abbas, M., Ben Haj Amara, A., Zubarev, A., \& Kuzhir, P. (2017). Twostage kinetics of field-induced aggregation of medium-sized magnetic nanoparticles. The Journal of chemical physics, 146(11), 114902.

Ezzaier, H., Marins, J. A., Claudet, C., Hemery, G., Sandre, O., \& Kuzhir, P. (2018). Kinetics of aggregation and magnetic separation of multicore iron oxide nanoparticles: effect of the grafted layer thickness. Nanomaterials, $8(8), 623$.

Gabayno JLF, Liu DW, Chang M, Lin YH. 2015 Controlled manipulation of Fe3O4 nanoparticles in an oscillating magnetic field for fast ablation of microchannel occlusion. Nanoscale 7, 3947-3953.

Gupta, A. K.; Gupta, M. Synthesis and Surface Engineering of Iron Oxide Nanoparticles For Biomedical Applications. Biomaterials 2005, 26, 3995-4021

Happel, J. B., \& Brenner, H. H (1981) Low Reynolds number hydrodynamics. Martinus Nijhoff Publishers, The Hagues.

Hassan, M. K., \& Hassan, M. Z. (2008). Condensation-driven aggregation in one dimension. Physical Review E, 77(6), 061404.

Ivanov, A. O., \& Zubarev, A. Y. (1998). Ostwald ripening kinetics in a magnetic fluid made metastable by a strengthening of an external magnetic field. Physical Review E, 58(6), 7517.

Jeffery, G. B. (1922). The motion of ellipsoidal particles immersed in a viscous fluid. Proceedings of the Royal Society of London. Series A, 102(715), 161-179.

Kalikmanov V.I. (2013) "Nucleation theory". Lecture notes in physics, v. 860. Springer Dordrecht

Kim, H., Sau, M., \& Furst, E. M. (2020). An expanded state diagram for the directed self-assembly of colloidal suspensions in toggled fields. Langmuir, 36, 33, 9926-9934.

Kolev, N. I. (1993). Fragmentation and coalescence dynamics in multiphase flows. Experimental Thermal and Fluid Science, 6(3), 211-251.

Kuzhir P., Raboisson-Michel M., Queiros Campos J., Verger-Dubois G. \&| Zubarev A. Yu (2020). Unified mathematical model of the kinetics of nanoparticle phase condensation in magnetic fields. Math Meth Appl Sci. 1-13; DOI: 10.1002/mma.6739

Kuzhir, P., López-López, M. T., \& Bossis, G. (2009). Magnetorheology of fiber suspensions. II. Theory. Journal of rheology, 53(1), 127-151.

Landau L. D. and E. M. Lifshitz (1984) Electrodynamics of Continuous Media. Pergamon, New York.

Landau L. D. and E. M. Lifshitz, Statistical Physics, 3rd ed., Vol. 1 (Pergamon, Oxford, 1980).

Laskar, J. M., Philip, J., \& Raj, B. (2010). Experimental investigation of magnetic-field-induced aggregation kinetics in nonaqueous ferrofluids. Physical Review E, 82(2), 021402.

Lebedev, A. V., Engel, A., Morozov, K. I., \& Bauke, H. (2003). Ferrofluid drops in rotating magnetic fields. New Journal of Physics, 5(1), 57.

Leulmi, S.; Chauchet, X.; Morcrette, M.; Ortiz, G.; Joisten, H.; Sabon, P.; Livache, T.; Hou, Y.; Carrière, M.; Lequien, S.; Dieny, B. Triggering the Apoptosis of Targeted Human Renal Cancer Cells by the Vibration of Anisotropic Magnetic Particles Attached to The Cell Membrane. Nanoscale 2015, 7, 15904-15914.

Levich, B, Physicochemical Hydrodynamics, Englewood Cliffs, N.J., Prentice-Hall, 1962 
Li Q, Liu X, Chang M, Lu Z. 2018 Thrombolysis enhancing by magnetic manipulation of Fe3O4 nanoparticles. Materials 11, 2313.

Lifschitz, E. M., and L. P. Pitajewski (1983). "Physical kinetics." Textbook of theoretical physics. 10. AkademieVerlag; Berlin.

Massart, R. (1981). Preparation of aqueous magnetic liquids in alkaline and acidic media. IEEE transactions on magnetics, 17(2), 1247-1248.

Mohapatra, D. K., \& Philip, J. (2019). Effect of surface charge screening on critical magnetic fields during field induced structural transitions in magnetic fluids. Journal of Applied Physics, 125(24), 244301.

Newman, D. M.; Heptinstall, J.; Matelon, R. J.; Savage, L.; Wears, M. L.; Beddow, J.; Cox, M.; Schallig, H. D. F. H.; Mens, P. F. A Magneto-Optic Route Toward the In Vivo Diagnosis of Malaria: Preliminary Results and Preclinical Trial Data. Biophys. J. 2008, 95, 994-1000.

Newman, D.; Matelon, R. Method and Device for Detecting an Analyte. U.S. Patent Application 14/241,534, May 7, 2015.

Perigo, E. A., Hemery, G., Sandre, O., Ortega, D., Garaio, E., Plazaola, F., \& Teran, F. J. (2015). Fundamentals and advances in magnetic hyperthermia. Applied Physics Reviews, 2(4), 041302.

Promislow, J. H., \& Gast, A. P. (1997). Low-energy suspension structure of a magnetorheological fluid. Physical Review E, 56(1), 642

Qian, J., \& Law, C. K. (1997). Regimes of coalescence and separation in droplet collision. Journal of fluid mechanics, 331, 59-80.

Roger, S., Talbot, D., \& Bee, A. (2006). Preparation and effect of Ca2+ on water solubility, particle release and swelling properties of magnetic alginate films. Journal of Magnetism and Magnetic Materials, 305(1), 221 227.

Sandre, O., Browaeys, J., Perzynski, R., Bacri, J. C., Cabuil, V., \& Rosensweig, R. E. (1999). Assembly of microscopic highly magnetic droplets: Magnetic alignment versus viscous drag. Physical Review E, 59(2), 1736.

Schrittwieser, S.; Pelaz, B.; Parak, W. J.; Lentijo-Mozo, S.; Soulantica, K.; Dieckhoff, J.; Ludwig, F.; Altantzis, T.; Bals, S.; Schotter, J. Homogeneous Protein Analysis by Magnetic Core-Shell Nanorod Probes. ACS Appl. Mater. Interfaces 2016, 8, 8893-8899.

Schrittwieser, S.; Pelaz, B.; Parak, W. J.; Lentijo-Mozo, S.; Soulantica, K.; Dieckhoff, J.; Ludwig, F.; Schotter, J. Direct Protein Quantification in Complex Sample Solutions By Surface-Engineered Nanorod Probes. Sci. Rep. 2017, 7, 4752.

Sherman, Z. M., \& Swan, J. W. (2016). Dynamic, directed self-assembly of nanoparticles via toggled interactions. ACS nano, 10(5), 5260-5271.

Sherman, Z. M., Rosenthal, H., \& Swan, J. W. (2018). Phase Separation Kinetics of Dynamically Self-Assembling Nanoparticles with Toggled Interactions. Langmuir, 34(3), 1029-1041.

Socoliuc V., Bica D. (2001) Experimental investigation of magneticinduced phase-separation kinetics in aqueous ferrofluids. In: Dékány I. (eds) Adsorption and Nanostructure. Progress in Colloid and Polymer Science, vol 117. Springer, Berlin, Heidelberg.

Socoliuc, V., Vékás, L., \& Turcu, R. (2013). Magnetically induced phase condensation in an aqueous dispersion of magnetic nanogels. Soft Matter, 9(11), 3098-3105.

Stikuts, A. P., Perzynski, R., \& Cēbers, A. (2020). Spontaneous order in ensembles of rotating magnetic droplets. Journal of Magnetism and Magnetic Materials, 500, 166304.

Swan, J. W., Bauer, J. L., Liu, Y., \& Furst, E. M. (2014). Directed colloidal self-assembly in toggled magnetic fields. Soft Matter, 10(8), 1102-1109.

Swan, J. W., Vasquez, P. A., Whitson, P. A., Fincke, E. M., Wakata, K., Magnus, S. H., F. De Winne, M. R. Barratt, J. H. Agui, R. D. Green,N. R. Hall, D. Y. Bohman, C.T. Bunnell, A. P. Gast, and E. M. Furst. (2012). Multi-scale kinetics of a field-directed colloidal phase transition. Proceedings of the National Academy of Sciences, 109(40), 16023-16028.

Szalai, I., Nagy, S., \& Dietrich, S. (2015). Linear and nonlinear magnetic properties of ferrofluids. Physical Review E, 92(4), 042314.

Tartaj, P., Morales, M. P., Veintemillas-Verdaguer, S., Gonzalez-Carreño, T., \& Serna, C. J. (2006). Synthesis, properties and biomedical applications of magnetic nanoparticles. Handbook of magnetic materials, 16(5), 403482. 
Van de Ven, T. G. (1989). Colloidal hydrodynamics. Academic press.

Vinod, S., \& Philip, J. (2018). Experimental evidence for the significant role of initial cluster size and liquid confinement on thermo-physical properties of magnetic nanofluids under applied magnetic field. Journal of Molecular Liquids, 257, 1-11.

Wang, B.; Bienvenu, C.; Mendez-Garza, J.; Lançon, P.; Madeira, A.; Vierling, P.; Di Giorgio, C.; Bossis, G. Necrosis of Hepg2 Cancer Cells Induced by the Vibration of Magnetic Particles. J. Magn. Magn. Mater. 2013, 344, 193-201.

Wilhelm, C., Gazeau, F., \& Bacri, J. C. (2003). Rotational magnetic endosome microrheology: Viscoelastic architecture inside living cells. Physical Review E, 67(6), 061908.

Zubarev, A. Y., \& Ivanov, A. O. (1997). Kinetics of a magnetic fluid phase separation induced by an external magnetic field. Physical Review E, 55(6), 7192.

Zubarev, A., Iskakova, L., López-López, M. T., Kuzhir, P., \& Bossis, G. (2014). On the theory of magnetoviscous effect in magnetorheological suspensions. Journal of rheology, 58(6), 1673-1692. 